\title{
A fluid-structure interaction solver for the study on a passively deformed fish fin with non-uniformly distributed stiffness
}

\author{
Yang Luo ${ }^{\mathrm{a}}$, Qing Xiao, ${ }^{\mathrm{a}, *}$, Guangyu Shi ${ }^{\mathrm{a}}, \mathrm{Li} \mathrm{Wen}^{\mathrm{b}}$, Daoyi $\mathrm{Chen}^{\mathrm{c}}$ and Guang Pan ${ }^{\mathrm{d}}$ \\ aDepartment of Naval Architecture, Ocean and Marine Engineering, University of Strathclyde, Glasgow, \\ G4 0LZ, United Kingdom
}

${ }^{\mathrm{b} S}$ School of Mechanical Engineering and Automation, Beihang University, Beijing 100191, People's Republic of China

'Division of Ocean Science and Technology, Graduate School at Shenzhen, Tsinghua University, Shenzhen 518055, People's Republic of China

${ }^{\mathrm{d} S}$ School of Marine Science and Technology, Northwestern Polytechnical University, Xi'an 710072, People's Republic of China

*Correspondent: qing.xiao@strath.ac.uk

\begin{abstract}
Research on fish locomotion has made extensive progress towards a better understanding of how fish control their flexible body and fin for propulsion and maneuvering. Although the biologically flexible fish fins are believed to be one of the most important features to achieve optimal swimming performance, due to the limitations of the existing numerical modeling tool, studies on a deformable fin with a non-uniformly distributed stiffness are rare. In this work, we present a fully coupled fluid-structure interaction solver which can cope with the dynamic interplay between flexible aquatic animal and the ambient medium. In this tool, the fluid is resolved by solving Navier-Stokes equations based on the finite volume method with a multi-block grid system. The solid dynamics is solved by a nonlinear finite element method. A sophisticated improved IQN-ILS coupling algorithm is employed to stabilize solution and accelerate convergence. To demonstrate the capability of the developed Fluid-Structure-Interaction solver, we investigated the effect of five different stiffness distributions on the propulsive performance of a caudal peduncle-fin model. It is shown that with a non-uniformly distributed stiffness along the surface of the caudal fin, we are able to replicate similar real fish fin deformation. Consistent with the experimental observations, our numerical results also indicate that the fin with a cupping stiffness profile generates the largest thrust and efficiency whereas a heterocercal flexible fin yields the least propulsion performance but has the best maneuverability.
\end{abstract}

\footnotetext{
* Author to whom correspondence should be addressed. Electronic mail: qing.xiao@strath.ac.uk. Tel: +44 01415484779
} 


\section{Introduction}

Fluid-structure interaction (FSI) is a common phenomenon in nature, in which the structure is subjected to the motion induced by the unsteady fluid forces, and in return, the flow around the structure is also affected by the structure response. Many engineering problems involve FSI phenomena, such as their applications in aerospace, ocean engineering, biomedical and civil engineering etc. One specific application in ocean engineering is the bio-inspired autonomous underwater vehicle (AUV) and robot. The advantages of such AUVs over the conventional vehicles which use rotational propellers are their low noise generation, the excellent maneuverability and high propulsion efficiency at low-speed cruising (Reddy $\mathrm{N}$ et al., 2018). The studies on the aquatic animal swimming mechanism have provided scientific insights for the design of those bio-inspired AUVs. Among various features of different swimmer locomotion, one of the most important features is its flexible body and/or caudal and pectoral fins interaction with the aquatic environment (Flammang and Lauder, 2008). The common flow features shared by such flexible swimmers are their complex three-dimensional and time-dependent structure deformation, leading to the intrinsic complexity of their surrounded fluid flow (Tian et al., 2014).

In the past studies, to understand the fundamental locomotion mechanism of the aquatic animals, the flexible fish fin or body were usually simplified as rigid models (Dong et al., 2006; Triantafyllou et al., 1993). More recently, some studies have investigated the flexible propulsors and their propulsion performance, which can be improved as compared to a rigid model. However, the so-called "flexibility" in these studies refers to a mathematically pre-defined fin deformation by reconstructing the realistic kinematics observed/recorded from the live fish experiment (Bozkurttas et al., 2009; Kern and Koumoutsakos, 2006; Wolfgang et al., 1999). Therefore, only the hydrodynamics response to the designated structure deformation was examined, whereas the effect of resultant fluid forces on the flexible fins was neglected.

On the other hand, recent anatomical studies indicated that fishes are characterized by a composite structure (Puri et al., 2018; Youngerman et al., 2014) instead of being made of uniform material as simplified in the previous studies (Dai et al., 2012b; Heathcote and Gursul, 2007; Yeh and Alexeev, 2016). The aforementioned simplifications may pose a significant impact on their predicted propulsion performance, which was proved by the studies (Shoele and Zhu, 2010) and (Shoele and Zhu, 2012), where the strengthened leading edge of fish pectoral fins achieved a higher propulsion efficiency as compared to a fin with uniformly distributed stiffness. This composite biological structure is also believed to be able to achieve an effective active and/or 
passive structure deformation pattern with multiple degrees of freedom, which could further result in the complex variations of fish's conformations during its maneuvering locomotion (Flammang and Lauder, 2008). A recent study by Flammang and Lauder (2009) found five movement patterns from the recorded three-dimensional kinematic data and high-speed video of a live bluegill sunfish caudal fin, and they were given the names of flat, cupping, W-shape, undulation and rolling motion respectively. It was illustrated that the curvature configurations contribute to fish's maneuvering behavior, e.g., a W-shape mode involves the kicking maneuvering. A similar phenomenon has also been observed by Youngerman et al. (2014) in a study of free-swimming ghost knifefish.

Generally, experimental and numerical modeling are the two approaches to elucidate the underlying mechanism of FSI phenomenon involving the flexible deformation of aquatic animals (Li et al., 2018). The experimental approach often investigates fish and fins locomotion through the observation of live fish and the measurement of their movement patterns, gait and elastic biological material properties (Alben et al., 2007; Flammang and Lauder, 2008; Kancharala and Philen, 2016; Taft and Taft, 2012). The obtained quantitative results manifested that the flexible fin shows a non-monotonous dependence of the bending rigidity along the proximal-distal axis.

The above studies with live fish experiment have some limitations due to the difficulties to control real fish and the other real-time experimental measurements (Lauder, 2015). An alternative way is to use a bio-inspired robotic fish to replicate the main features of a real fish. For example, four passively-flexing fish-like foil models were designed by Lucas et al. (2015) to examine the effects of foil non-uniform stiffness on the swimming performance. Their results indicated that the two foils with anterior regions of high stiffness and posterior regions of low stiffness outperform the foils with uniform distribution of flexibility in terms of self-propelled speeds and thrust production. In the study of Kancharala and Philen (2016), the stiffness profiles of a real fish fin were measured by using digital image correlation (DIC) techniques. With those experimental extracted data, a robotic fin with a chordwise variation of stiffness was fabricated to study the locomotion performance in a water tunnel. Similar to the observations achieved by Lucas et al. (2015), their results showed that the fins with varying stiffness generated larger thrusts and higher efficiency compared to the uniform ones. This conclusion is reinforced by a recent publication by Reddy $\mathrm{N}$ et al. (2018) on a flat plate fin with trapezoidal geometry.

Although in the above-mentioned experiments, biomimetic models can be constructed with different physical material, they are still subjected to the availability of material diversity for 
conducting a systematic parametric study. Additionally, some key hydrodynamics parameters (e.g., surface force and structural stress of fish) remain less known in an experimental work. To compensate for these weaknesses associated with experiment testing, numerical simulation plays a role in presenting holographic flow information, as it enables to answer "what if" type questions, which makes it more attractive in comparison with experimental studies. Especially thanks to the high-performance computation and high-fidelity numerical techniques, computational modeling becomes an indispensable approach along with experiments in biomimetic research.

With some sophisticated simulation modeling, a few researchers have conducted numerical studies on flexible fish fin. A series of work has been done by Zhu and Shoele (2008). In their early work, a fully coupled fluid-structure interaction tool was developed which combined a nonlinear Euler-Bernoulli beam model and a boundary-integral hydrodynamics model. The tool was then used to investigate fish pectoral fin during labriform swimming. Two different flexible fins were considered, i.e., one with monotonous structural properties while another with a leading edge rigidity strengthened. They found that the fin strengthening enhanced the performance by reducing the effective angle of attack as well as decreasing the power expenditure (Shoele and Zhu, 2010). Due to the limitations of their hydrodynamics model derived from a potential flow theory, their studies failed to resolve the complicated flow separation and fin vortex evolution, while such phenomena often dominate in fish swimming and thus has a significant impact on the performance estimation. A recent improvement was made using the immersed boundary method (IBM) to take into account the viscous effect (Shoele and Zhu, 2012, 2013). Nonetheless, these models are all two-dimensional. Tian et al. (2014) coupled a three-dimensional viscous fluid solver with a nonlinear finite-element solid-mechanics solver and applied this to the simulations of the aerodynamics of the flexible flapping wings and the flow-induced vocal fold vibration. These case studies demonstrated the versatility of this method. Nevertheless, the advanced, efficient and accurate FSI solvers are still very few.

In this study, we proposed a fully coupled FSI model to tackle the aforementioned FSI challenges for a three-dimensional passively deformed fish fin with a non-uniform distribution stiffness along the fin surface. Within this coupling framework, the hydrodynamics around the fin is simulated by solving unsteady Navier-Stokes equations with a cell-centered finite-volume method (Liu et al., 2016), while its structural mechanics is resolved using a finite element code CalculiX (Dhondt, 2004). These two solvers are implicitly coupled in a partitioned approach via preCICE (Bungartz et al., 2016). Although FSI studies on underwater flexible bodies are not scarce, the existing FSI models are often limited to simple structural mechanics, such as either 2D problems 
or linear elasticity. Distinct from those methods, with a finite element method and abundant elements types, the present structure solver is able to analyze a much wider range of nonlinear structures which may involve complex material behaviors along with large displacement and complex deformation. Additionally, thanks to the sophisticated and robust coupling algorithms implemented in preCICE, this new FSI solver enables to model strong coupling interaction between fish fin and its immersed aquatic environment more efficiently. For example, an elaborate coupling algorithm, the Interface Quasi-Newton Inverse Least Squares method (IQNILS) (Degroote et al., 2009; Haelterman et al., 2016; Mehl et al., 2016), is applied in this work to stabilize solution and accelerate convergence, which is rare in most of existing partitioned coupling models in biomimetic studies.

By applying the developed fully coupled FSI solver, we focus on the study of a caudal fin with complex variations of flexural rigidity in this work. It is inspired by the experimental study by Esposito et al. (2012) and a recent numerical study by Zhu and Bi (2017). They both investigated the propulsion performance of a caudal fin involving its non-uniform stiffness and complex deformation patterns. In the study of Zhu and Bi (2017), they employed a boundary-element mothed for the hydrodynamic analysis, where the vortices shed from the fin and viscous shear layer were ignored, which may lead to the loss of accuracy. Moreover, the fin surface was modeled as a simplified linear spring, and therefore, the deformation of their predicted flexible fin surface appeared not smooth, inconsistent with the smooth curvature fashion observed from real fish. It should also be mentioned that the viscous effect and vorticity shedding from the locations, which is not limited to the trailing edge were also considered in our recent paper (Shi et al., 2019). However, the model was geometrically simplified as a rectangular plate composed of several flexural rays connected by linear springs. Thus, a continuously passive deformation of the flexible fin was not well replicated. In this work, we will demonstrate the robustness of the developed FSI tool by solving some generalized FSI problems and then take a flexible fish fin as an example of application. It will serve as a framework for the future bio-inspired studies involving active and passive control associated with complex structure material.

The remainder of this paper is organized as follows: the geometry, structural properties and kinematics of the flexible caudal peduncle-fin model is introduced in Section 2. We also define the performance metrics in this section. In the next section, the governing equations of fluids and solids are presented. Section 4 presents the numerical approaches used to solve the fluid-structure coupled problem. In Section 5, several validations are conducted and sensitivity studies are 
demonstrated. The results of the numerical studies are presented in Section 6. Finally, the conclusions are drawn in Section 7.

\section{Problem Formulations}

The current peduncle and caudal fin model is inspired by the experiment test conducted by Esposito et al. (2012), where a robotic fish tail mimicking the locomotion of the Bluegill Sunfish (Lepomis macrochrus) has been experimentally examined. In their experiment, the robot consists of a rigid peduncle and a flexible caudal fin, and more details for the experimental setup can be found in (Esposito et al., 2012).

However, it is worthwhile to note that the current study does not attempt to duplicate the real fish in terms of its lifelike geometry or material features in-vivo. Instead, like the studies by Esposito et al. (2012) and Zhu and Bi (2017), we focus on some main characteristics, e.g., anisotropic flexibility and associated fluid-structure interplay, extracted from a real fish fin. Fig. 1 illustrates the geometry and dimensions of the peduncle and caudal fin model. The thickness $h$ of the model is $0.02 c$, where $c$ is the chord length of the fin at the midline. All the edges are chamfered to ease our CFD mesh generation.

The kinematics of the present model is described as follows. The peduncle combined with the caudal fin rotate harmonically around the $z$-axis with the reference point $\boldsymbol{O}$ in a uniform flow in the positive $x$-direction with a velocity of $U_{\infty}$. The time-dependent pitch motion of the model is described by $\theta(t)=\theta_{m} \sin (2 \pi f t)$, where $\theta_{m}$ is the peak amplitude and $f$ denotes the oscillating frequency.

The dimensionless parameters are defined as: the Reynolds number $\operatorname{Re}=U_{\infty} c / v$; mass ratio $m^{*}=\rho_{s} h / \rho_{f} c$; the reduced frequency $f^{*}=f_{c} / U_{\infty}$; the Poisson ratio the $v_{s}$; dimensionless stiffness $K=E I /\left(\rho_{f} U_{\infty}^{2} c^{3}\right)$, where $E$ denotes Young's modulus, $I=h^{3} / 12$ is the area moment of inertia of the cross section.

\subsection{Structural model of the caudal fin - the complex variations of stiffness}

Structurally, this fin is composed of 19 segments (typically, the number of rays of a real fish fin ranges from 10 to 20, (Westneat et al., 2004)), as shown in Fig. 2(a). To replicate various fin deformation patterns observed in (Esposito et al., 2012) and (Zhu and Bi, 2017), each segment is assigned with a unique $K$, i.e., for $i$ th segment, the normalized flexural rigidity is 
$K_{i}=E_{i} I / \rho_{f} U_{\infty}^{2} c^{3}(i=1, \ldots, N)$, where $N=19$. Five variation patterns of $K$ corresponding to different deformation fashions are considered in this work:

1. Uniform distribution: $K_{i}=K_{c}$.

2. Cupping distribution: $K_{i}=K_{c} R_{i} / R$, where $R_{i}=1+\gamma\left[1-\sin \frac{\pi(i-1)}{N-1}\right]$.

3. Reverse cupping distribution: $K_{i}=K_{c} R_{i} / R$, where $R_{i}=1+\gamma \sin \frac{\pi(i-1)}{N-1}$.

4. W-shape distribution: $K_{i}=K_{c} R_{i} / R$, where $R_{i}=1+\gamma\left[1-\left|\sin \frac{2 \pi(i-1)}{N-1}\right|\right]$.

5. Heterocercal distribution: $K_{i}=K_{c} R_{i} / R$, where $R_{i}=1+\gamma\left[1-\sin \left(\frac{\pi|i-N|}{2(N-1)}\right)\right]$.

Here, $R=\frac{1}{N} \sum_{i=1}^{N} R_{i}$, and $K_{c}$ is a constant and used to quantify the mean flexibility of all the fin segments. The ratio of the stiffness between the least flexible segment and the most flexible one is determined by the parameter $\gamma$. In this simulation, $\gamma=10$ is selected. The variations pattern of flexural rigidity is demonstrated in Fig. 2(b).

\subsection{Performance metrics}

To evaluate the propulsion performance of the caudal fin, the instantaneous thrust and power expenditure coefficients are defined as

$$
C_{T}=-\frac{F_{x}}{\frac{1}{2} \rho_{f} U_{\infty}^{2} S}, C_{P}=-\frac{M_{o} \dot{\theta}}{\frac{1}{2} \rho_{f} U_{\infty}^{3} S}
$$

where $F_{x}$ is the total hydrodynamic forces on the caudal fin in the $x$-direction, $S$ is the reference area, i.e., the area of the fin in $x z$ plane, $M_{o}$ represents the $z$-component of the reaction moment at point $\boldsymbol{O}$. Meanwhile, the lateral force is defined as the hydrodynamic forces in the $y$-direction, as well as lift force in the $z$-direction 


$$
C_{y}=\frac{F_{y}}{\frac{1}{2} \rho_{f} U^{2} S}, C_{z}=\frac{F_{z}}{\frac{1}{2} \rho_{f} U^{2} S} .
$$

The propulsion efficiency $\eta$ is given by

$$
\eta=\frac{\bar{C}_{T}}{\bar{C}_{P}}
$$

where $\bar{C}_{T}$ and $\bar{C}_{P}$ are the time-averaged values of $C_{T}$ and $C_{P}$ within one oscillating period. It is noted that Froude efficiency proposed by Tytell and Lauder (2004) was defined to evaluate the swimming efficiency by many studies (Borazjani and Sotiropoulos, 2008; Liu et al., 2017). But it can only be applied when the mean value of net force acting on the swimmer body is zero which does not apply to this work (Borazjani and Sotiropoulos, 2008), therefore, it is not defined in this study.

\section{Governing Equations}

In this section, the governing equations for the fluid and the solid are outlined. The fluid and structural domain are represented by $\Omega_{f}$ with the boundary $\Gamma_{f}$ and $\Omega_{s}$ with $\Gamma_{s}$ respectively. The fluid-structure interface $\Gamma_{i}=\Gamma_{f} \cap \Gamma_{s}$ between the fluids and structures is the common boundary of the two domains. The information at the interface is exchanged between the fluid and structural solver.

\subsection{Fluid solver}

The in-house fluid solver solves the viscous, compressible flow which is governed by the laws of the conservation of mass, momentum and energy, and it can be expressed in the integral form as

$$
\frac{\partial}{\partial t} \iiint_{\Omega_{,}} \mathbf{W} d \Omega_{f}+\oiint_{\Gamma_{,}}\left(\mathbf{F}_{c}-\mathbf{F}_{d}\right) \cdot \mathbf{n} d \Gamma_{f}=0
$$

where $\mathbf{n}$ is the unit normal vector in the outward direction. The conservative variable vector $\mathbf{W}$ in Eq.(4) is defined as

$$
\mathbf{W}=\{\rho, \rho u, \rho v, \rho w, \rho E\}^{T},
$$


where $\rho$ is the density of the fluid, $u, v, w$ represent the three velocity components in Cartesian coordinates and $E$ is the total energy.

The vector $\mathbf{F}_{c}$ in Eq.(4) are the convective and pressure fluxes which are given by

$$
\mathbf{F}_{c}=\left[\begin{array}{ccc}
\rho u_{r} & \rho v_{r} & \rho w_{r} \\
\rho u u_{r}+p & \rho u v_{r} & \rho u w_{r} \\
\rho v u_{r} & \rho v v_{r}+p & \rho v w_{r} \\
\rho w u_{r} & \rho w v_{r} & \rho w w_{r}+p \\
\rho E u_{r}+p u & \rho E v_{r}+p v & \rho E w_{r}+p w
\end{array}\right]
$$

where $p$ is the pressure. Within an FSI application, to allow for the movement and deformation of the domain boundary, the arbitrary Lagrangian-Eulerian (ALE) formulation is the most commonly used to handle the flow equations on a deformation mesh. It is achieved here by defining fluxes relative to the motion of the surfaces of the control volume, which are expressed by the relative velocity $u_{r}, v_{r}$ and $w_{r}$ given as

$$
\begin{aligned}
& u_{r}=u-u_{g} \\
& v_{r}=v-v_{g} \\
& w_{r}=w-w_{g},
\end{aligned}
$$

where the flow velocity $\{u, v, w\}^{T}$ and the grid velocity $\left\{u_{g}, v_{g}, w_{g}\right\}^{T}$ are described in a stationary Cartesian coordinate system.

The fluxes arising from the viscous shear stresses and thermal diffusion are denoted by $\mathbf{F}_{d}$ and can be formulated as

$$
\mathbf{F}_{d}=\left[\begin{array}{ccc}
0 & 0 & 0 \\
\tau_{x x} & \tau_{x y} & \tau_{x z} \\
\tau_{y x} & \tau_{y y} & \tau_{y z} \\
\tau_{z x} & \tau_{z y} & \tau_{z z} \\
u \tau_{x x}+v \tau_{x y}+w \tau_{x z}-q_{x} & u \tau_{y x}+v \tau_{y y}+w \tau_{y z}-q_{y} & u \tau_{z x}+v \tau_{z y}+w \tau_{z z}-q_{z}
\end{array}\right]
$$

where, for a Newton-Fourier fluid considered here, the shear stresses $\tau_{\alpha \beta}$ are defined as 


$$
\tau_{\alpha \beta}=\mu\left[\left(\frac{\partial u_{\alpha}}{\partial x_{\beta}}+\frac{\partial u_{\beta}}{\partial x_{\alpha}}\right)-\frac{2}{3}(\nabla \cdot \mathbf{u}) \delta_{\alpha \beta}\right], \alpha, \beta \in\{x, y, z\}
$$

The heat fluxes $\boldsymbol{q}$ are defined as

$$
\boldsymbol{q}=-k \nabla T
$$

where $k$ denotes the thermal conductivity and $T$ is the temperature.

\subsection{Structural solver}

For the present FSI applications in which the temperature is assumed to be known, the basic equation of the finite element method is the weak form of the balance of momentum which can be written in the differential form as

$$
\rho_{s} \frac{D^{2} \mathbf{U}}{D^{2} t^{2}}=\nabla \cdot \mathbf{P}+\rho_{s} \mathbf{f}
$$

where the acceleration of the material point is obtained by the second derivatives of displacement vector $\mathbf{U}$ of the structure, and surface forces are modeled by the second Piola-Kirchoff stress tensor $\mathbf{P}$ and body forces of per unit mass such as gravity are represented by $\mathbf{f}$. The solid density is denoted by $\rho_{s}$.

A constitutive equation describing the relation between the stress and the strain is used to close up Eq.(11). For a Saint Venant-Kirchhoff material, the second Piola-Kirchoff stress tensor $\mathbf{P}$ is obtained by

$$
\mathbf{P}=\mathbf{C}: \mathbf{E}, \quad \mathbf{E}=\frac{1}{2}\left(\mathbf{F}^{\mathrm{T}} \mathbf{F}-\boldsymbol{\delta}\right)
$$

where $\mathbf{C}$ is the elasticity tensor, $\mathbf{E}$ represents the Green-Lagrange strain tensor, the deformation gradient is characterized by $\mathbf{F}$ and $\boldsymbol{\delta}$ is the unit tensor.

\section{Numerical Approach}

In this section, the numerical techniques applied to solve the governing equations of fluid and structure and the coupling algorithm are described. A partitioned or segregated approach is 
implemented to handle FSI coupling in the present work. The governing equations for the fluid and the structure are solved separately and the coupling is established externally.

\subsection{Fluid dynamics}

In this work, the governing equations of flow, i.e., Eq.(4), are discretized using a cell-centered finite volume method based on a multi-block structured grid system. Using a structured methodology, the fluid domain $\Omega_{f}$ is divided into an array of hexahedral cells. For each cell indexed by $(i, j, k)$ Eq.(4) holds and can be reformulated in the semi-discrete form given by

$$
\frac{\partial}{\partial t}\left(\mathbf{W}_{i, j, k} \Delta \Omega_{f}\right)_{i, j, k}-\mathbf{R}_{i, j, k}=0
$$

where $\mathbf{W}_{i, j, k}$ are the average flow variables of the cell, $\Delta \Omega_{f}$ denotes the cell volume, and $\mathbf{R}_{i, j, k}$ is the residual, which measures the net fluxes entering the hexahedral cell through all six cell faces. To stabilize the scheme and eliminate the spurious numerical oscillations, an artificial dissipative term is introduced in $\mathbf{R}_{i, j, k}$ (Jameson et al., 1981).

Through the application of the dual-time stepping scheme (Jameson, 1991), Eq.(13) can be reformulated at each time step as the following steady-state flow problem in a pseudo-time $t^{*}$

$$
\frac{\partial}{\partial t^{*}} \mathbf{W}^{(n+1)}=\frac{1}{\Delta \Omega_{f}} \mathbf{R}^{*}\left(\mathbf{W}^{(n+1)}\right)
$$

where

$$
\mathbf{R}^{*}\left(\mathbf{W}^{(n+1)}\right)=\mathbf{R}\left(\mathbf{W}^{(n+1)}\right)-\frac{3\left(\mathbf{W} \Delta \Omega_{f}\right)^{(n+1)}-4\left(\mathbf{W} \Delta \Omega_{f}\right)^{(n)}+\left(\mathbf{W} \Delta \Omega_{f}\right)^{(n-1)}}{2 \Delta t},
$$

where the solution vectors of two previous time levels are used here which are denoted by superscript $n$ and $n-1$.

In this study, parallelization is achieved by domain decomposition based on message passing interface (MPI) to enable large-scale computation. The different grid blocks are automatically distributed over a number of processors by the block size with the application of a load-balancing algorithm. Furthermore, the local time-stepping and multigrid method is implemented to accelerate the convergence and implicit residual smoothing is applied to increase the stability of the solution. 


\subsection{Solid dynamics}

The general governing equation of the solid dynamics, i.e. Eq.(11), is discretized using the finite element method. Using the standard virtual work method, the linear algebraic equation system can be obtained by discretizing Eq.(11) in the whole solid domain $\Omega_{s}$ as

$$
[\mathbf{K}]\{\mathbf{U}\}+[\mathbf{M}] \frac{D^{2}}{D t^{2}}\{\mathbf{U}\}=\{\mathbf{F}\}
$$

where $[\mathbf{K}]$ is the global stiffness matrix, $[\mathbf{M}]$ is the global matrix and $[\mathbf{F}]$ is the global force vector respectively. The three assembled terms are given by

$$
\begin{aligned}
& {[\mathbf{K}]:=\sum_{e}[\mathbf{L}]_{e}^{T}[\mathbf{K}]_{e}[\mathbf{L}]_{e},} \\
& {[\mathbf{M}]:=\sum_{e}[\mathbf{L}]_{e}^{T}[\mathbf{M}]_{e}[\mathbf{L}]_{e},} \\
& \{\mathbf{F}\}:=\sum_{e}[\mathbf{L}]_{e}^{T}\{\mathbf{F}\}_{e},
\end{aligned}
$$

where $[\mathbf{K}]_{e}$ denotes the element stiffness matrix, $[\mathbf{M}]_{e}$ is the element mass matrix, $\{\mathbf{F}\}_{e}$ is the element force vector and $[\mathbf{L}]_{e}$ denotes the element localization matrix.

The time domain is discretized using the $\alpha$-method here. Denoting the velocity vector $\{\mathbf{V}\}:=\{\dot{\mathbf{U}}\}$ and acceleration vector $\{\mathbf{A}\}:=\{\ddot{\mathbf{U}}\}$, the solution at time level $n+1$ can be obtained by

$$
\begin{gathered}
\{\mathbf{V}\}_{n+1}=\{\tilde{\mathbf{V}}\}_{n+1}+\gamma \Delta t\{\mathbf{A}\}_{n+1}, \\
\{\mathbf{U}\}_{n+1}=\{\tilde{\mathbf{U}}\}_{n+1}+\beta(\Delta t)^{2}\{\mathbf{A}\}_{n+1},
\end{gathered}
$$

where $\{\tilde{\mathbf{V}}\}_{n+1}$ and $\{\tilde{\mathbf{U}}\}_{n+1}$ can be considered as the predictor at time level $n+1$ which are only dependent on the values at time level $n$. A more detailed interpretation of its implementation can be found in Appendix A.

In this work, the implementation of finite element method solver is based on CalculiX written by Dhondt (2004), in which a variety of element types including the brick element, the tetrahedral element and the wedge element are used to discretize the solid domain and define the shape functions. 


\subsection{Mesh deformation algorithm}

In FSI simulation, the structure is subject to the deformation under the surface normal force applied to it. The configuration of the interface $\Gamma_{i}$ changes with time and the flow grid has to be updated to accommodate with the unsteady boundary. An efficient algebraic method is applied to interpolate the displacements of the meshes at the interface $\Gamma_{i}$ to yield displacements of the inner grid points of the fluid domain $\Omega_{f}$ by iteratively solving the equations of static equilibrium with a predictor-corrector process (Tsai et al., 2001). Hereby, all the block corner points of the fluid domain are assumed to be connected with each other by springs whose rigidities are inversely proportional to the length of the connecting edge. Once the displacements of vertices at the boundary are determined, the displacements of inner points are obtained using a spring-analogy method (Batina, 1990) and arc-length-based Trans-Finite Interpolation (TFI) method (Sadeghi et al., 2003). To retain the original grid quality after deformation, Hermite polynomials are used to maintain the grid angles of the original grid near the wall.

\subsection{Fluid-structure coupling}

\subsubsection{Coupling scheme}

In the present study, a partitioned coupling scheme is used to reduce the effort to adapt the original computation codes and preserve both the advanced features of the fluid and solid solvers. However, the partitioned method does not mean it is a loose coupling, in which numerical instability poses challenges to convergence (Förster et al., 2007; Küttler and Wall, 2008). Especially, in the current FSI application for a bio-inspired fish fin propulsion, the densities of fluid and solid are comparable, thus the numerical instabilities must be carefully dealt with (Causin et al., 2005).

Within the present framework of a partitioned coupling, to ensure the aforementioned numerical stability during the present FSI simulation, an implicit scheme is designed here. In this work, the in-house fluid solver is integrated with CalculiX via preCICE (Bungartz et al., 2016), and the adapter of CalculiX used to connect it with preCICE is implemented by Uekermann et al. (2017).

Two implicit coupling fashions, i.e., parallel and serial which correspond to the parallel and staggered execution of the fluid and solid solver respectively, are implemented in preCICE (Mehl et al., 2016). Here, to take serial as an example, the simplified flow chart illustrating the serial implicit coupling is presented in Fig. 3. A more detailed description of the flow chart of Fig. 3 can be found in Appendix B. Within the current coupling framework, an improved IQN-ILS 
method (Degroote et al., 2009) implemented in preCICE, is applied to stabilize and accelerate the coupling iterations. The input and output data of previous iterations are used to approximate the inverse Jacobian of the residual operator of the fixed-point formulation and perform a Newtonlike solver step (Mehl et al., 2016). In addition, to avoid the potential singularity of the approximated inverse Jacobian, a QR-based filtering technique is employed to filter out data which is linearly dependent (Haelterman et al., 2016). In the present work, a parallel coupling is mainly employed for better efficiency, despite that both lead the same physical results which has been demonstrated in (Mehl et al., 2016).

\subsubsection{Data mapping and communication}

The fluid and structural meshes are non-matching here, which makes it impossible to copy the data values from one side to another directly. Therefore, an interpolation method is necessary to map data between two solvers at the interface. In this work, the radial basis functions (RBF) based interpolation as described in (Lindner et al., 2017) is applied to transfer node forces from the fluid to the solid and vertices displacements oppositely. Both the conservative and consistent mappings are implemented in the RBF interpolation. We apply a consistent mapping during the transformation of displacements, while a conservative fashion for force mapping which makes the sum of the data values in both sides equal to ensure the energy balance over the interface (de Boer et al., 2008).

The communication between the fluid and structural solver is a fully parallel point-to-point fashion. In this study, it means that the communication channels are only constructed between those processors that have been assigned with blocks with boundaries interface which are marked after the distribution of grid blocks.

\subsubsection{Convergence criterion}

A crucial ingredient of the partitioned coupling is the measurement of its convergence towards the monolithic solution in each time step. By applying the discrete $l_{2}$ norm of the difference of displacements between the current and last iteration, a scalar representative of the residual vector can be obtained as

$$
\left\|\boldsymbol{r}_{k+1}\right\|_{2}=\left(\sum_{i}\left(r_{k+1, i}\right)^{2}\right)^{1 / 2} .
$$

Therefore, a relative convergence measure yields as 


$$
\frac{\left\|\boldsymbol{r}_{s, k+1}\right\|_{2}}{\left\|\boldsymbol{s}_{k}\right\|_{2}}<\varepsilon \wedge \frac{\left\|\boldsymbol{r}_{f, k+1}\right\|_{2}}{\left\|\boldsymbol{f}_{k}\right\|_{2}}<\varepsilon,
$$

where $\varepsilon$ is predefined. With the proceeding of iterations the relative residual drops. If it is not below than $\varepsilon$, another sub-iteration will be performed again until the relative residual small enough to satisfy the criterion.

\section{Validations and Self-consistency Study}

The fluid solver used in the present work has been extensively validated in our previous work (Liu et al., 2013; Liu et al., 2016; Xiao and Liao, 2010; Xiao et al., 2012). Here the following three properly selected cases are used to validate the coupling of the fluid solver with CalculiX via preCICE. Additionally, a self-consistency study is performed to provide a sensitivity study on the new FSI solver.

\subsection{Validation cases}

\subsubsection{Flow over a flexible cantilever behind a square cylinder}

This case consists of a fixed square bluff body with an elastic cantilever attaching in its wake (Nakata and Liu, 2012; Olivier et al., 2009; Wood et al., 2010). Previous studies indicated that the flow separated from the leading edge of the square cylinder would induce a periodic oscillation of the flexible cantilever.

The layout of the computational domain and generated fluid mesh are presented in Fig. 4. In this simulation, the fluid domain contains 153428 cells in the form of a multi-block grid, while structural mesh comprises 123 quadratic wedge elements with standard shape functions (Dhondt, 2003).

In the present simulation, $\operatorname{Re}=U d / v=330$, based on the dimension $d=0.01 \mathrm{~m}$ of the square body, which can give rise to a transient Von Karman vortex street under such a Reynolds number.

The physical properties, i.e. mass ratio $m^{*}=\rho_{s} e / \rho_{f} l=1.27$, non-dimensional bending stiffness $K=E I /\left(\rho_{f} U^{2} l^{3}\right)=0.23$, and Poisson's ratio $v_{s}=0.35$ are chosen to make the frequency of shedding vortex approximate the first Eigen-frequency of the cantilever, so that a remarkable oscillation can be observed. In the structural part, the left end of the cantilever is set as fixed, i.e., the $x$ and $y$ direction freedom is locked, and the right end is set free. 
Fig. 5 depicts the displacement of the free end of the flexible beam in the y-direction with two time step sizes, which are defined as $\Delta t=\Delta t U / d$. As seen, the displacement lies in a range of $0.85 \sim 1.30 \mathrm{~cm}$, and dimensionless oscillation period $\overline{\mathrm{T}}=\mathrm{T} U / d$ varies around 17.08 equivalent to a dimensionless frequency $\bar{f}=1 / \overline{\mathrm{T}}=0.0585$. It is very close to the theoretical Eigen-frequency of the flexible cantilever, which is 0.0591. Observed from published literature (Dettmer and Perić, 2006; Habchi et al., 2013; Matthies and Steindorf, 2003; Olivier et al., 2009; Wood et al., 2010), the displacement amplitude lies between $0.8 \sim 1.4 \mathrm{~cm}$, and $\overline{\mathrm{T}}$ ranges between 15.80 and 17.44. Therefore, the present simulation results have a good agreement with the previous numerical solution.

The vorticity contour in the $z$-direction within one oscillation period is presented in Fig. 6. During one oscillation period, two clock-wise vortices form at the upper region while another two counterparts form at the lower region. In addition, the Von Karman vortex street behind the square bluff is dispersed due to the existence of oscillating elastic cantilever. Then it seems to be stretched along the downstream direction, but evolves to an independent round-similar shape away from the appendix.

\subsubsection{The bending of a $3 D$ flexible plate in a uniform incoming flow}

This validation involves a flexible plate which is bent while placed in crossflow. The original case derives from an experimental study on the flow-induced reconfiguration of flexible aquatic vegetation conducted by Luhar and Nepf (2011). In their experiment, drag and posture of the model blades that span the natural range for stiffness and buoyancy of sea-grass were measured and recorded. One of their experiment cases was then numerically simulated as an FSI validation by Tian et al. (2014). In their work, they quantitatively compared the results with experimental data in the presence of gravity and buoyancy and performed a series of simulations in the absence of them for the purpose of benchmarks studies. Here, the latter cases are chosen to validate our proposed multi-physics numerical suite.

The configuration of the elastic plate is depicted in Fig. 7, which is placed vertically in the crossflow direction with its bottom end clamp-mounted but the end free. The dimensionless parameters are: length $h=5 b$, thickness $t=0.2 b$, where $b$ is the width, $\operatorname{Re}=U_{0} h / v=100$, $m^{*}=\rho_{s} b / \rho_{f} t=0.14, K=E I / \rho_{f} U_{0}^{2} b^{3}=2.39, v_{s}=0.4$. The fluid computational domain is a rectangle box extending from $(-5 b,-8 b,-8 b)$ to $(12 b, 8 b, 8 b)$ with a number of 3916111 calculation 
grid cells, whose origin locates at the center of the plate. The first grid height is $0.002 b$. The nondimensional time step size $\Delta t=0.0208 U_{0} / b$. Meanwhile, 1400 quadratic brick elements (Dhondt, 2004) with standard shape functions are used to model the flexible plate in CalculiX.

During the simulation, the flexible plate eventually reaches a stable configuration, as shown in Fig. 8. As seen, the flow incident surface of the plate near the bottom end bears the largest fluid pressure, while with approaching the top side, its hydrodynamic pressure experiences a continuous decrease, as well as the pressure difference between the two surfaces in the flow direction. This is consistent with the experimental data that in comparison with a rigid aquatic vegetation model, a flexible one tends to bend to a steady configuration to reduce the flowinduced drag (Luhar and Nepf, 2011; Tian et al., 2014).

The displacement of the plate center and the drag coefficient, which is defined as $C_{d}=F_{x} /\left(0.5 \rho_{f} U_{0}^{2} b h\right)$, in the absence of gravity and buoyancy, are compared in Table 1 . It indicates that the present FSI simulation results match well with the counterparts in (Tian et al., 2014).

\subsubsection{The response of a flexible plate in a forced harmonic heave motion}

This numerical validation case involves an experimental study conducted by Paraz et al. (2014); (Paraz et al., 2016). It consists of a horizontal flexible plate, which is made of the polysiloxane. The plate has a rounded leading edge and a tapered trailing edge. The thickness of plate $e$ is $0.004 \mathrm{~m}$, chord length $c$ is $0.12 \mathrm{~m}$ and span $s$ is $0.12 \mathrm{~m}$, giving an aspect ratio $s / c=1$. In their experiment, the leading edge was forced into a harmonic heave motion while the trailing edge was set free. The elastic plate deformed under the hydrodynamic forces (Paraz et al., 2014).

The CFD simulation is carried out with a structured fluid mesh which contains 57424 hexahedron cells along with 105 structural quadratic brick elements. The response of the plate is characterized by the change of the relative displacement of the trailing edge with respect to that of the leading edge $A_{\mathrm{TE}} / A_{\mathrm{LE}}$ and the phase difference $\varphi$ as the forcing frequency is varied. The response of the plate with varied forcing frequency obtained by our numerical simulations is depicted in Fig. 9.

It can be found from Fig. 9 that the current emulation results agree well with the counterparts in the experiment. A sharp peak is observed in the displacement curves of the trailing edge when the forcing frequency approximates the natural frequency $f_{0}$ in both experimental and numerical simulation, where the $A_{\mathrm{TE}}$ is 2.5 times larger than the $A_{\mathrm{TE}}$. This is the first resonance peak 
according to the analysis by Paraz et al. (2014). With the increase of forcing frequency, the phase shift $\varphi$ witnesses a continuous increase, which indicates stronger interactions between fluid and structure with a larger forcing frequency.

In Fig. 10, the deformation of the plate at the resonance frequency is illustrated by the superposition of the centerlines of the plate within one forcing cycle. According to the definition of Dai et al. (2012b), the current deformation pattern of the plate corresponds to the first mode. It is noted that a weak asymmetry in up-and-down of the shape mode is observed in Fig. 10(a) from the experimental results, but not from that of the numerical simulations results. This may be explained by the fact that in the experiment, the density of the plate is slightly larger than that of water and the gravity effect plays a role in the response of the plate, while in the present simulation the gravity influence is ignored. Even so, since the two densities approximate to each other, the neglect of the gravity does not lead a big difference.

\subsection{Self-consistency study}

The self-consistency study is performed to assess the appropriate mesh and time-step resolution for $\operatorname{Re}=U_{\infty} c / v=2500, m^{*}=0.02, v_{s}=0.25, f^{*}=1, \theta_{m}=10$ degree, and the fin is uniformly distributed with a dimensionless stiffness of $K_{c}=1$. The computational domain and fluid mesh around the caudal peduncle-in model are shown in Fig. 11. On the peduncle-fin surface, the noslip condition is applied, while for the other boundaries, the non-reflective far-field boundary condition is imposed. Three grids are generated: a coarse grid with 2294292 nodes and minimum spacing of $1.48 \times 10^{-3} c$ in each direction, a medium grid with 4032768 nodes and minimum spacing of $9.9 \times 10^{-4} c$, and a fine grid with 5791680 nodes and minimum spacing of $6.19 \times 10^{-4} c$. The structural mesh contains 1461 quadratic wedge elements. Furthermore, the non-dimensional time-step corresponding to the coarse grid is $\Delta t=\Delta t / T=0.00909$, where $T$ is the oscillation period, the medium grid is $\Delta t=0.00694$ and the time step size for the fine one is $\Delta t=0.00556$. The results of $C_{T}$ when three different girds and time step sizes are used are shown in Fig. 12, and the mean coefficients $\overline{C_{T}}, \overline{C_{p}}$ as well as efficiency are compared in Table 2. Observed from the comparison, we find that the medium resolution setup is sufficient to simulate the flow field around the caudal fin. Therefore, the medium grid with a time-step $\Delta t=0.00694$ is used for the following simulations. 


\section{Results}

With the above code verifications and resolution study, we applied the developed FSI solver to the flexible fins study aforementioned. The Reynolds number under consideration is $\operatorname{Re}=2500$, the mass ratio is $m^{*}=0.02$, the rotation angle $\theta_{m}$ is 10 degree, the Poisson ratio $v_{s}$ is 0.25 , and the reduced frequency is $f^{*}=1$. Most of these parameters are chosen to match with that in the experimental study by Ren et al. (2016a) at similar scales. It is worth noting that under such relatively low Reynolds number, no turbulence model is employed in our study, i.e., the flow is assumed as laminar, which is adopted in biomimetic studies (Mittal et al., 2006) (Liu et al., 2017; Shoele and Zhu, 2012). For all the simulations, the relative coupling convergence criterions $\varepsilon_{\text {relative }}$ of displacement and fluid forces within each time step are set as $3 \times 10^{-3}$ to ensure the energy balance at the interface.

The predicted time-averaged thrust, lateral forces, lift forces, power input coefficients and efficiency, as well as the tail excursion at point $A$ of the caudal fin under various flexural stiffnesses are summarized in Fig. 13, in which the values of a rigid fin are also included to facilitate comparison. As seen from the figure, the flexibility and its distribution have a significant effect on the propulsive performance and its deformation of the caudal fin. Overall, for all the cases considered here, the tail excursion increases monotonically as $K_{c}$ is raised, with the exception of that of the fin with a heterocercal profile when $K_{c}$ is larger than 10. Meanwhile, the $\overline{C_{T}}, \overline{C_{y}}, \overline{C_{z}}, \overline{C_{P}}$ and $\eta$ vary remarkably when the caudal fin is assigned with different stiffness distributions along the surface of fins. In addition, even with the same $K_{c}$, fins with different stiffness distributions present diverse propulsive capabilities. In particular, the heterocercal profile, the only asymmetrical distribution, shows distinct features as compared to others in terms of the curve variation patterns, which is consistent with the observations from the experiment work by Esposito et al. (2012).

Compared with a rigid fin, flexible fins yield larger thrust unless when the stiffness is very small, i.e., the fin is too compliant, and under this condition, the excursion of the flexible fin is also smaller than that of a rigid one. Under the parameters studied, the thrusts generated by flexible fins firstly witness a sharp increase, and most of them reach the peaks when $K_{c}=5$, with an exception for heterocercal distribution, where it crests at $K_{c}=10$. This is consistent with the findings by Esposito et al. (2012), where the mean fluid forces generated by both asymmetric and 
symmetric motions peak at different fin stiffness. After the crest, all the $\overline{C_{T}}$ decease with the increase of stiffness. This variation pattern can be found in many previous FSI studies involving bio-inspired propulsion (Dai et al., 2012a; Olivier and Dumas, 2016; Shi et al., 2017). Generally, among these five variations, $\overline{C_{T}}$ produced by a fin with heterocercal stiffness distribution differs most with others, and apart from when the fin is highly flexible, its magnitude is much smaller than the others. Meanwhile, the fin with cupping stiffness profile generates the largest thrust and these with uniform and $\mathrm{W}$-shape distribution produce quite close thrust values, which is consistent with the results in (Esposito et al., 2012).

With a closer inspection of Fig. 13(b), we can find that when $K_{c}<5$, as the stiffness is increased, the differences of thrust generation due to the various flexibility distributions become more pronounced, which is consistent with the conclusion drawn by Esposito et al. (2012). In addition, for much stiffer fins, i.e., $K_{c}>10$, the differences appear less distinct. This may be attributed to the fact that with the fins become stiffer, the deformations are more limited, and thus diminishing the differences caused by various stiffness profiles. This has been reflected by other research on the flexible swimmer studies (Dai et al., 2012b; Olivier and Dumas, 2016). However, previous numerical studies (Shi et al., 2019; Zhu and Bi, 2017) concluded that the differences become more remarkable as the fins are more flexible, which seems contradictory with the present results and the experimental study. Nevertheless, it is noted that fundamental difference exists in terms of kinematics and numerical model to calculate the deformation of fins. In their studies, the locomotion is accomplished by the sway motion of rays embedded in the fin membrane instead of the rotation in the experiment (Esposito et al., 2012) and this work. On the other hand, in their fin models, the various stiffness profiles are assigned on each fin ray, i.e., nonlinear Euler-Bernoulli beam and the deformation of the fin is mainly determined by simplified linear spring models between rays. In this study, the fin is composed of segments with different rigidities, and the flexing is determined both by the fluid forces and assigned stiffness.

In terms of the propulsion efficiency $\eta$, clear peaks are observed for all the variation patterns of stiffness with the increase of flexural rigidities $K_{c}$. The highest efficiency is achieved by the fin with cupping inflexibility distribution at $K_{c}=1.5$. Similar to the variation patterns of $\overline{C_{T}}, \eta$ of the fin with a cupping stiffness profile performs best, and those of the fins with uniform and Wshape distribution show no evident difference. Again, the fin with a heterocercal stiffness fashion yields the lowest efficiency like the smallest thrust shown in Fig. 13(b) among these five variation 
patterns when $K_{c}$ remains the same. In general, the efficiency is more sensitive to the variation of $K_{c}$ when its values are small, which can be observed that when the $K_{c}$ is small, e.g., when it is less than 1 , the $\eta$ curves drop sharply, and even a slight decrease of $K_{c}$ results in a significant loss in propulsion efficiency. Instead, as the fin is much less flexible, the variation of $\eta$ appears flatter, which is consistent with previous results in (Shi et al., 2019; Zhu and Bi, 2017).

The propulsion efficiency is determined both by $\overline{C_{T}}$ and $\overline{C_{P}}$, therefore, the curves of timeaveraged energy expenditure coefficients may explain the above observed variation fashions. Seen from Fig. 13(c), apart from the $\overline{C_{P}}$ of the fin with a heterocercal stiffness profile when $K_{c}$ is larger than 2, the five curves present no pronounced difference in magnitude. This may manifest the reason for the similarity of variations in $\overline{C_{T}}$ and $\eta$, i.e., when $\overline{C_{P}}$ approach with each other, the variation of $\eta$ is mainly determined by $\overline{C_{T}}$. On the other hand, by comparison of Fig. 13(b) and (c), we may find that difference of the propulsion efficiency among the fin with heterocercal and other stiffness distributions mainly results from its less thrust production.

Regarding the time-averaged lateral forces coefficients, more complex variations are observed compared with the discussion above for $\overline{C_{T}}$ and $\overline{C_{P}}$ indicated by Fig. 13(b) and (c). Under the parameters studies, much larger lateral forces are produced by flexible fins in comparison with that by a rigid fin, and the $\overline{C_{y}}$ curves present clear groove-similar profile. Large lateral force may not be beneficial to a straight-line cruising for a fish, but it can provide better maneuvering via providing a turning moment. It can be observed in Fig. 13(e) that, unsurprisingly, the heterocercal distribution, as the only asymmetrical stiffness fashion, yields the largest mean lift forces, having the same magnitude as thrust, while $\overline{C_{z}}$ of fins with the other distribution patterns are negligible, which is in accordance with the experimental observation from Esposito et al. (2012).

The $\overline{C_{z}}$ of the fin with a heterocercal profile are all negative, indicating their contribution to the downwards maneuvering, and it witnesses a significant increase and then declines as the increase of stiffness. This may seem contradictory to the results of Zhu and Bi (2017), where they concluded that the lift forces which were all positive experienced a continuous drop when the fin is stiffer. However, it should be noted that in this study, the heterocercal profile differs from that in (Zhu and $\mathrm{Bi}, 2017)$, i.e., the flexural rigidity increases from ventral to dorsal as shown in Fig. 2(b), which is inverse to their counterparts. This may explain the resultant opposite lift direction. 
On the other hand, different variation patters are likely to be attributed to the aforementioned differences such as kinematics and different numerical model and solution of deformation, combined with an inviscid fluid model which neglects the viscous effect and vortices effect shed from the leading dorsal and ventral edge.

To evaluate the swimming performance of aquatic animals, a dimensionless parameter, the Strouhal number, is widely used in biomimetic studies to quantify the propulsion of a pitch locomotor (Eloy, 2012; Sfakiotakis et al., 1999; Triantafyllou et al., 1993). Here we defined it as $S t=f a / U_{\infty}$, with $a$ the maximum peak-to-peak excursion of the tail. In Fig. 14, we re-plotted the $\overline{C_{T}}, \overline{C_{P}}$ and $\eta$ against the St. As depicted in Fig. 14, under the parameters studied, the $S t$ number ranges between 0.3 and 0.8 . In most cases, with the increase of the $S t, \overline{C_{T}}$ and $\overline{C_{P}}$ witness continuous rise for all the five stiffness variation patterns. It can be also observed that the peak efficiency typically occurs within a range of 0.4 to 0.6 , which is in accordance with the results in (Dai et al., 2012b) of a flexible pitching plate.

The instantaneous thrust, lateral forces and power expenditure coefficients when $K_{c}=5$ are depicted in Fig. 15. The other symmetric stiffness profiles present similar fashions as cupping which is shown in this figure. In accordance with the results in (Esposito et al., 2012), the magnitude of the thrust of flexible fins increases as the fin accelerates from its extreme lateral position to the midline, and then reaches the largest approximately at the mid-stroke. Thrust gradually decreases after the fins pass the midline and then turns into a net drag, which is consistent with previous results (Akhtar et al., 2007; Zhu and Shoele, 2008). In terms of $C_{y}$, with a comparison of Fig. 13(d) and Fig. 15(b), even though the mean lateral force is smaller for a rigid fin, however, the curves of flexible fins are flatter, which means less disturbance from lateral forces and it benefits a steady straight line swimming for locomotors. Compared with a rigid fin, flexible fins require less power input, which may be explained by the fact that the flexibility reduces the work done to the surrounding fluid significantly.

The typical deformation patterns of fins with various stiffness fashions are presented in Fig. 16. For better visualization of the curvatures in the spanwise direction, Fig. 17 depicts the surface patterns of trailing edge in the $y-z$ plane during one flapping period. To facilitate the comparison, the posterior views of the real fish in-vivo obtained in the experimental studies using high-speed digital video cameras (Flammang and Lauder, 2008; Flammang and Lauder, 2009) are also presented. In their experiment, the fish was placed in a flow tank and induced to swim freely 
within the center region of the flow tank. By comparison of Fig. 16 with Fig. 17, it can be observed that the pure passive deformations of our models by assigning non-uniform stiffness distributions cannot exactly replicate as pronounced spanwise deformations as observed from a real fish fin. This difference may be explained by the fact that fish can actively control the propulsive surface conformation by complex muscle activities at the bases of the fish ray halves (hemitrichs) as suggested by Lauder and Madden (2006), and this active control may play a dominant role in the fin deformation. With a closer inspection of Fig. 17, it can be found that one common feature of the deformation styles of our models is that the deflection around the center of the fin is smaller than those away from the central segment regardless of how the stiffness distributes. This deformation pattern was also observed by Zhu and Shoele (2008) in a raystrengthened caudal fin study. This is because the deflection of a cantilever, the flexible segment herein which composes the fin surface, is entirely determined by the external fluid load, its structural stiffness and the segment length.

The displacements of the point $A$ and $B$ at the trailing edge of the caudal fin are shown in Fig. 18 . In general, it can be observed that the point $A$ always leads the excursion. Like the propulsive performance presented in Fig. 13, the displacements of $A$ and $B$ of the fin with uniform and Wshape stiffness style show no evident distinction. The excursions of $A$ and $B$ for a heterocercal distribution present significant difference with each other, which is caused by the large deflection of the dorsal fin segment. It can also be observed that for a highly flexible fin, the deflection in the $y$-direction is rather small, which is directly associated with the actual pitching angle of the peduncle-fin model with respect to the $x$-axis. With the increase of inflexibility, the actual pitching angle increases accordingly. This may explain the thrust enhancement depicted in Fig. 13(b) when $K_{c}=5$ compared with that of fins that are more compliant.

Fig. 19 presents the wake flows of the caudal peduncle-fin in $Y$ vorticity contour. Two main tip vortices shed from the dorsal- and ventral-most trailing edge of the fin are formed parallel and alternatively. They have opposite rotation directions with one in counterclockwise and the other clockwise, and for symmetrical stiffness profile, their vortices are approximately equal. These results match with those obtained in (Esposito et al., 2012; Ren et al., 2016b) using digital particle image velocimetry (DPIV) techniques and previous numerical simulations (Shi et al., 2019). A closer inspection of Fig. 19(e) reveals that another two observable pairs of small vortices are also attached right behind the trailing edge of the fin with cupping stiffness style, and away from that, the farther wake vortices are funneled inward towards the midline. As observed by Esposito et al. (2012), this funneling causes an accelerated jet of fluid between the vortices. These additional 
small vortices and remarkable funneling effect may contribute to the best propulsive performance of cupping distribution among the five profiles. However, in comparison, the asymmetrical distribution of vortices breaks the funneling as well as the increase of fluid velocity in the wake of the fin with heterocercal stiffness distribution, which deteriorates its thrust generation.

The wake structures of the flexible fins with the cupping and heterocercal stiffness distribution when $K_{c}=2$ are depicted in Fig. 20. It can be observed that the wake of the fins is composed of a sequence of vortex rings which induce a jet flow in the forward thrust generation direction. The cow-horn shaped vortex rings associated with a cupping stiffness profile present a perfect symmetry relative to the midline in the $z$-direction. Similar wake patterns are generated by the fins with a symmetric stiffness distribution, i.e., uniform, W-shape and reverse cupping style, therefore, they are not presented here. For a heterocercal stiffness style, as the $Y$ vorticity shown in Fig. 19(b), the vortex rings show a distinctive asymmetry with regard to the midline, which induces the flow to point slightly downwards (or upwards). Therefore, prominent lift forces are produced as depicted in Fig. 13(e).

The pressure distribution along the fin surface is depicted in Fig. 21. With an exception of the fin with a heterocercal stiffness profile, which is asymmetric in both left and right sides, the others are all symmetric in the $z$-direction. The right side, as the incident flow surface, is covered by negative pressure, which is applicable to symmetric stiffness variation patterns. However, the pressure distribution on the left side shows that a cupping profile fin has the largest high-pressure zone thus yields the largest pressure difference. This prominent pressure difference along the negative $x$-direction contributes to the thrust generation as indicated by the largest thrust shown in Fig. 13(b). Due to the power input are almost identical for four symmetric stiffness profiles as displayed in Fig. 13(c), a maximum or optimal efficiency is achieved with a cupping fin.

\section{Conclusions}

In this work, we developed a CFD numerical solver to simulate the fluid-structure interaction problem in aquatic propulsion, which is achieved via a coupling of our in-house fluid code with a finite element method based structural solver. Within this framework, the surrounding flow of a compliant fin is resolved by the solution of three-dimensional unsteady Navier-Stokes equations. The structural response is obtained by solving the equations of momentum balance in the weak form with a finite element method. A sophisticated IQN-ILS coupling algorithm is employed to stabilize numerical solution and accelerate iterative convergence. 
Through three properly selected validation cases, we demonstrate the accuracy of the developed code via comparisons with other available numerical or experimental results. Beyond that, we employ this multi-physics solver to simulate a rotational passively deformed caudal fin model with the non-uniform flexibility distribution along the fin surface. The fin consists of nineteen segments with variable stiffness. In an attempt to test whether the CFD tool is possible to replicate the real fin deformation, five stiffness distribution patterns are considered, e.g., uniform, heterocercal, W-shape, reverse cupping and cupping.

The obtained numerical modeling results are consistent with the experiment from Esposito et al. (2012) in two aspects. Firstly, we illustrate that the fin deformation, particularly the trailing edge curvature resembles the experimental observation. Secondly, the fin with cupping deformation

yields the best overall performance, whereas fin with heterocercal profile, representing the rolling motion of fish, produces the least thrust but with considerable lift forces for maneuvering purpose. The excellent performance brought by fin with cupping stiffness is due to the aforementioned remarkable funneling effect and the attached small vortices around trailing edges. However, this does not occur with a heterocercal pattern. We also find that with the increase of flexural rigidities, the difference in terms of thrust generation becomes more pronounced before the flexibility reaches a threshed.

As a final point, it is worthwhile to mention that, in the present study, we consider a flexible fin shape with a passively deformed feature. However, the developed numerical solver is not limited to such simple geometry. Also, with the use of this tool, it is proved that we can achieve an equivalent surface deformation and an enhanced propulsion effect as a biologically actively muscle-controlled flexible fish fin. Therefore, the potential applications of this tool can be found in wide bio-mimetic areas such as AUV and aerial vehicle.

\section{Acknowledgements}

This work used the Cirrus UK National Tier-2 HPC Service at EPCC (http://www.cirrus.ac.uk) funded by the University of Edinburgh and EPSRC (EP/P020267/1). We acknowledge Dr. Benjamin Uekermann at the Eindhoven University of Technology for his kind comments and suggestions for this study. The first author would like to thank the China Scholarship Council (CSC) for financial support during his study in the United Kingdom. 


\section{Appendix A.}

The velocity vector at time level $n$ is given by

$$
\begin{gathered}
\{\tilde{\mathbf{V}}\}_{n+1}=\{\mathbf{V}\}_{n}+(1-\gamma) \Delta t\{\mathbf{A}\}_{n}, \\
\{\tilde{\mathbf{U}}\}_{n+1}=\{\mathbf{U}\}_{n}+\Delta t\{\mathbf{V}\}_{n}+\frac{1}{2}(\Delta t)^{2}(1-2 \beta)\{\mathbf{A}\}_{n} .
\end{gathered}
$$

By applying Newton's Second Law of Motion, the acceleration vector $\{\mathbf{A}\}_{n+1}$ in Eq.(18) and Eq.(19) can be obtained by solving the following equation

$$
\left[\mathbf{M}^{*}\right]\{\mathbf{A}\}_{n+1}=\left\{\mathbf{F}^{*}\right\}
$$

where

$$
\begin{gathered}
{\left[\mathbf{M}^{*}\right]=[\mathbf{M}]+(1+\alpha) K \beta(\Delta t)^{2},} \\
\left\{\mathbf{F}^{*}\right\}=(1+\alpha)\{\mathbf{F}\}_{n+1}-\alpha\{\mathbf{F}\}_{n}-(1+\alpha) \mathbf{K} \tilde{\mathbf{U}}_{n+1}+\alpha \mathbf{U}_{n} .
\end{gathered}
$$

It is proved that if $\beta$ and $\gamma$ satisfy

$$
\beta=\frac{1}{4}(1-\alpha)^{2}, \gamma=\frac{1}{2}-\alpha
$$

the $\alpha$-method considered yields second-order accuracy and is unconditionally stable for $\alpha \in[-1 / 3,0]$ (Dhondt, 2004). The maximum high-frequency dissipation arises if $\alpha=-1 / 3$, while when $\alpha=0$, there is no high-frequency dissipation and the $\alpha$-method reduces to the Newmark algorithm. Here, in this study, the default value in CalculiX -0.05 is used.

\section{Appendix B.}

Following the flow chart of Fig. 3, at the very beginning of the time step $n$, the internal state of the current time step, i.e., the flow variables $\mathbf{W}$ and grid coordinates $\mathbf{x}$, are saved, which will be reloaded if current sub-iteration fails to converge. This serves to retain the same residual equation in every sub-iteration to approximate the aforementioned inverse Jacobian (Mehl et al., 2016). Within one time step, the structural solution $s_{k}^{n}$ at the interface, i.e., the displacements of the 
vertices at the boundaries in coupling iteration $k$, which has been post-processed using the aforementioned IQN-ILS scheme, is transformed to the fluid solver in a consistent mapping approach. With the displacements, the spring-analogy and TFI method based grid smooth technique are performed to update the inner fluid grid. After the solution of the flow governing equations in the current sub-iteration, the fluid forces $\tilde{\boldsymbol{f}}_{k}^{n}$ are derived from the pressure and the viscous shear stress of the interface. They are post-processed as $f_{k}^{n}$ and then transformed to the structural solver in a conservative mapping approach. Here, the fluid forces are calculated based on each cell surface on the coupling boundaries, but represented by a node fashion.

Through the above fixed-point iteration of the solution at the interface, the implicit coupling ensures the dynamic equilibrium and attempts to regain the solution of a monolithic system.

\section{Reference}

Akhtar, I., Mittal, R., Lauder, G.V., Drucker, E., 2007. Hydrodynamics of a biologically inspired tandem flapping foil configuration. Theoretical and Computational Fluid Dynamics 21, 155-170. Alben, S., Madden, P.G., Lauder, G.V., 2007. The mechanics of active fin-shape control in rayfinned fishes. Journal of the Royal Society, Interface 4, 243-256.

Batina, J.T., 1990. Unsteady Euler airfoil solutions using unstructured dynamic meshes. AIAA Journal 28, 1381-1388.

Borazjani, I., Sotiropoulos, F., 2008. Numerical investigation of the hydrodynamics of carangiform swimming in the transitional and inertial flow regimes. Journal of experimental biology 211, 1541-1558.

Bozkurttas, M., Mittal, R., Dong, H., Lauder, G.V., Madden, P., 2009. Low-dimensional models and performance scaling of a highly deformable fish pectoral fin. Journal of Fluid Mechanics 631, 311-342.

Bungartz, H.-J., Lindner, F., Gatzhammer, B., Mehl, M., Scheufele, K., Shukaev, A., Uekermann, B., 2016. preCICE-a fully parallel library for multi-physics surface coupling. Computers \& Fluids 141, 250-258.

Causin, P., Gerbeau, J.F., Nobile, F., 2005. Added-mass effect in the design of partitioned algorithms for fluid-structure problems. Computer Methods in Applied Mechanics and Engineering 194, 4506-4527.

Dai, H., Luo, H., Doyle, J.F., 2012a. Dynamic pitching of an elastic rectangular wing in hovering motion. Journal of Fluid Mechanics 693, 473-499.

Dai, H., Luo, H., Sousa, P.J.S.A.F.d., Doyle, J.F., 2012b. Thrust performance of a flexible lowaspect-ratio pitching plate. Physics of Fluids 24, 101903.

de Boer, A., van Zuijlen, A.H., Bijl, H., 2008. Comparison of conservative and consistent approaches for the coupling of non-matching meshes. Computer Methods in Applied Mechanics and Engineering 197, 4284-4297.

Degroote, J., Bathe, K.-J., Vierendeels, J., 2009. Performance of a new partitioned procedure versus a monolithic procedure in fluid-structure interaction. Computers \& Structures 87, 793-801. Dettmer, W., Perić, D., 2006. A computational framework for fluid-structure interaction: finite element formulation and applications. Computer Methods in Applied Mechanics and Engineering 195, 5754-5779. 
Dhondt, G., 2003. CalculiX CrunchiX USER'S MANUAL version 1.8. Mathematics Department, Macquarie University, Sydney.

Dhondt, G., 2004. The finite element method for three-dimensional thermomechanical applications. John Wiley \& Sons.

Dong, H., Mittal, R., Najjar, F., 2006. Wake topology and hydrodynamic performance of lowaspect-ratio flapping foils. Journal of Fluid Mechanics 566, 309-343.

Eloy, C., 2012. Optimal Strouhal number for swimming animals. Journal of Fluids and Structures 30, 205-218.

Esposito, C.J., Tangorra, J.L., Flammang, B.E., Lauder, G.V., 2012. A robotic fish caudal fin: effects of stiffness and motor program on locomotor performance. The Journal of Experimental Biology 215, 56-67.

Flammang, B.E., Lauder, G.V., 2008. Speed-dependent intrinsic caudal fin muscle recruitment during steady swimming in bluegill sunfish, Lepomis macrochirus. Journal of Experimental Biology 211, 587-598.

Flammang, B.E., Lauder, G.V., 2009. Caudal fin shape modulation and control during acceleration, braking and backing maneuvers in bluegill sunfish, Lepomis macrochirus. Journal of Experimental Biology 212, 277-286.

Förster, C., Wall, W.A., Ramm, E., 2007. Artificial added mass instabilities in sequential staggered coupling of nonlinear structures and incompressible viscous flows. Computer methods in applied mechanics and engineering 196, 1278-1293.

Habchi, C., Russeil, S., Bougeard, D., Harion, J.-L., Lemenand, T., Ghanem, A., Valle, D.D., Peerhossaini, H., 2013. Partitioned solver for strongly coupled fluid-structure interaction.

Computers \& Fluids 71, 306-319.

Haelterman, R., Bogaers, A.E.J., Scheufele, K., Uekermann, B., Mehl, M., 2016. Improving the performance of the partitioned QN-ILS procedure for fluid-structure interaction problems: Filtering. Computers \& Structures 171, 9-17.

Heathcote, S., Gursul, I., 2007. Flexible Flapping Airfoil Propulsion at Low Reynolds Numbers. AIAA Journal 45, 1066-1079.

Jameson, A., 1991. Time dependent calculations using multigrid, with applications to unsteady flows past airfoils and wings, 10th Computational Fluid Dynamics Conference. American Institute of Aeronautics and Astronautics.

Jameson, A., Schmidt, W., Turkel, E.L.I., 1981. Numerical solution of the Euler equations by finite volume methods using Runge Kutta time stepping schemes, 14th Fluid and Plasma Dynamics Conference. American Institute of Aeronautics and Astronautics.

Kancharala, A.K., Philen, M.K., 2016. Optimal chordwise stiffness profiles of self-propelled flapping fins. Bioinspiration \& Biomimetics 11, 056016.

Kern, S., Koumoutsakos, P., 2006. Simulations of optimized anguilliform swimming. Journal of Experimental Biology 209, 4841-4857.

Küttler, U., Wall, W.A., 2008. Fixed-point fluid-structure interaction solvers with dynamic relaxation. Computational mechanics 43, 61-72.

Lauder, G.V., 2015. Fish Locomotion: Recent Advances and New Directions. Annual Review of Marine Science 7, 521-545.

Lauder, G.V., Madden, P.G., 2006. Learning from fish: kinematics and experimental hydrodynamics for roboticists. International journal of automation and computing 3, 325-335.

Li, R., Xiao, Q., Liu, Y., Hu, J., Li, L., Li, G., Liu, H., Hu, K., Wen, L., 2018. A multi-body dynamics based numerical modelling tool for solving aquatic biomimetic problems.

Bioinspiration \& biomimetics 13, 056001.

Lindner, F., Mehl, M., Uekermann, B., 2017. Radial basis function interpolation for black-box multi-physics simulations, VII International Conference on Computational Methods for Coupled Problems in Science and Engineering, pp. 1-12. 
Liu, G., Ren, Y., Dong, H., Akanyeti, O., Liao, J.C., Lauder, G.V., 2017. Computational analysis of vortex dynamics and performance enhancement due to body-fin and fin-fin interactions in fish-like locomotion. Journal of Fluid Mechanics 829, 65-88.

Liu, W., Xiao, Q., Cheng, F., 2013. A bio-inspired study on tidal energy extraction with flexible flapping wings. Bioinspiration \& biomimetics 8, 036011.

Liu, W., Xiao, Q., Zhu, Q., 2016. Passive Flexibility Effect on Oscillating Foil Energy Harvester. AIAA Journal 54, 1172-1187.

Lucas, K.N., Thornycroft, P.J., Gemmell, B.J., Colin, S.P., Costello, J.H., Lauder, G.V., 2015. Effects of non-uniform stiffness on the swimming performance of a passively-flexing, fish-like foil model. Bioinspiration \& biomimetics 10, 056019.

Luhar, M., Nepf, H.M., 2011. Flow-induced reconfiguration of buoyant and flexible aquatic vegetation. Limnology and Oceanography 56, 2003-2017.

Matthies, H.G., Steindorf, J., 2003. Partitioned strong coupling algorithms for fluid-structure interaction. Computers \& Structures 81, 805-812.

Mehl, M., Uekermann, B., Bijl, H., Blom, D., Gatzhammer, B., van Zuijlen, A., 2016. Parallel coupling numerics for partitioned fluid-structure interaction simulations. Computers \& Mathematics with Applications 71, 869-891.

Mittal, R., Dong, H., Bozkurttas, M., Lauder, G., Madden, P., 2006. Locomotion with flexible propulsors: II. Computational modeling of pectoral fin swimming in sunfish. Bioinspiration \& biomimetics 1, S35.

Nakata, T., Liu, H., 2012. A fluid-structure interaction model of insect flight with flexible wings. Journal of Computational Physics 231, 1822-1847.

Olivier, M., Dumas, G., 2016. A parametric investigation of the propulsion of 2D chordwiseflexible flapping wings at low Reynolds number using numerical simulations. Journal of Fluids and Structures 63, 210-237.

Olivier, M., Morissette, J.-F., Dumas, G., 2009. A Fluid-Structure Interaction Solver for NanoAir-Vehicle Flapping Wings, 19th AIAA Computational Fluid Dynamics. American Institute of Aeronautics and Astronautics.

Paraz, F., Eloy, C., Schouveiler, L., 2014. Experimental study of the response of a flexible plate to a harmonic forcing in a flow. Comptes Rendus Mécanique 342, 532-538.

Paraz, F., Schouveiler, L., Eloy, C., 2016. Thrust generation by a heaving flexible foil: Resonance, nonlinearities, and optimality. Physics of Fluids 28, 011903.

Puri, S., Aegerter-Wilmsen, T., Jaźwińska, A., Aegerter, C.M., 2018. In vivo quantification of mechanical properties of caudal fins in adult zebrafish. The Journal of Experimental Biology 221, jeb171777.

Reddy N, S., Sen, S., Har, C., 2018. Effect of flexural stiffness distribution of a fin on propulsion performance. Mechanism and Machine Theory 129, 218-231.

Ren, Z., Hu, K., Wang, T., Wen, L., 2016a. Investigation of Fish Caudal Fin Locomotion Using a Bio-Inspired Robotic Model. International Journal of Advanced Robotic Systems 13, 87.

Ren, Z., Yang, X., Wang, T., Wen, L., 2016b. Hydrodynamics of a robotic fish tail: effects of the caudal peduncle, fin ray motions and the flow speed. Bioinspiration \& biomimetics 11, 016008.

Sadeghi, M., Yang, S., Liu, F., Tsai, H., 2003. Parallel computation of wing flutter with a coupled Navier-Stokes/CSD method, 41st Aerospace Sciences Meeting and Exhibit, p. 1347.

Sfakiotakis, M., Lane, D.M., Davies, J.B.C., 1999. Review of fish swimming modes for aquatic locomotion. IEEE Journal of oceanic engineering 24, 237-252.

Shi, G., Qing, X., Qiang, Z., Wei, L., 2019. Fluid-structure interaction modeling on a 3D raystrengthened caudal fin. Bioinspiration \& Biomimetics 14, 036012.

Shi, G., Xiao, Q., Zhu, Q., 2017. A Study of 3D Flexible Caudal Fin for Fish Propulsion, ASME 2017 36th International Conference on Ocean, Offshore and Arctic Engineering. American Society of Mechanical Engineers, pp. V07AT06A052-V007AT006A052. 
Shoele, K., Zhu, Q., 2010. Numerical simulation of a pectoral fin during labriform swimming. The Journal of Experimental Biology 213, 2038-2047.

Shoele, K., Zhu, Q., 2012. Leading edge strengthening and the propulsion performance of flexible ray fins. Journal of Fluid Mechanics 693, 402-432.

Shoele, K., Zhu, Q., 2013. Performance of a wing with nonuniform flexibility in hovering flight. Physics of Fluids 25, 041901.

Taft, N.K., Taft, B.N., 2012. Functional implications of morphological specializations among the pectoral fin rays of the benthic longhorn sculpin. The Journal of Experimental Biology 215, 27032710.

Tian, F.-B., Dai, H., Luo, H., Doyle, J.F., Rousseau, B., 2014. Fluid-structure interaction involving large deformations: 3D simulations and applications to biological systems. Journal of Computational Physics 258, 451-469.

Triantafyllou, G.S., Triantafyllou, M., Grosenbaugh, M., 1993. Optimal thrust development in oscillating foils with application to fish propulsion. Journal of Fluids and Structures 7, 205-224.

Tsai, H.M., F. Wong, A.S., Cai, J., Zhu, Y., Liu, F., 2001. Unsteady Flow Calculations with a Parallel Multiblock Moving Mesh Algorithm. AIAA Journal 39, 1021-1029.

Tytell, E.D., Lauder, G.V., 2004. The hydrodynamics of eel swimming: I. Wake structure. Journal of Experimental Biology 207, 1825-1841.

Westneat, M.W., Thorsen, D.H., Walker, J.A., Hale, M.E., 2004. Structure, function, and neural control of pectoral fins in fishes. IEEE Journal of Oceanic Engineering 29, 674-683.

Wolfgang, M.J., Anderson, J.M., Grosenbaugh, M.A., Yue, D.K., Triantafyllou, M.S., 1999. Near-body flow dynamics in swimming fish. The Journal of Experimental Biology 202, 2303 2327.

Wood, C., Gil, A.J., Hassan, O., Bonet, J., 2010. Partitioned block-Gauss-Seidel coupling for dynamic fluid-structure interaction. Computers \& Structures 88, 1367-1382.

Xiao, Q., Liao, W., 2010. Numerical investigation of angle of attack profile on propulsion performance of an oscillating foil. Computers \& Fluids 39, 1366-1380.

Xiao, Q., Liao, W., Yang, S., Peng, Y., 2012. How motion trajectory affects energy extraction performance of a biomimic energy generator with an oscillating foil? Renewable Energy 37, 61 75.

Yeh, P.D., Alexeev, A., 2016. Effect of aspect ratio in free-swimming plunging flexible plates. Computers \& Fluids 124, 220-225.

Youngerman, E.D., Flammang, B.E., Lauder, G.V., 2014. Locomotion of free-swimming ghost knifefish: anal fin kinematics during four behaviors. Zoology 117, 337-348.

Zhu, Q., Bi, X., 2017. Effects of stiffness distribution and spanwise deformation on the dynamics of a ray-supported caudal fin. Bioinspiration \& biomimetics 12, 026011.

Zhu, Q., Shoele, K., 2008. Propulsion performance of a skeleton-strengthened fin. Journal of Experimental Biology 211, 2087-2100. 
(a)

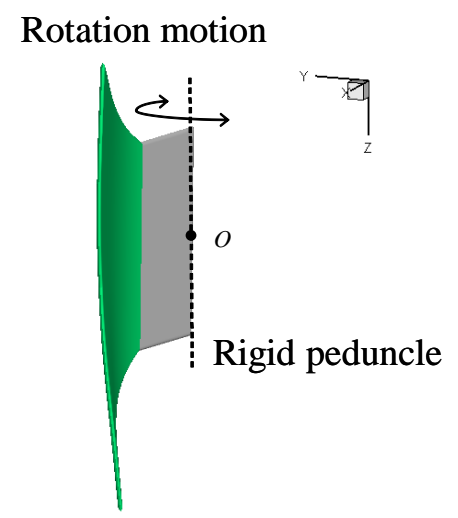

(b)

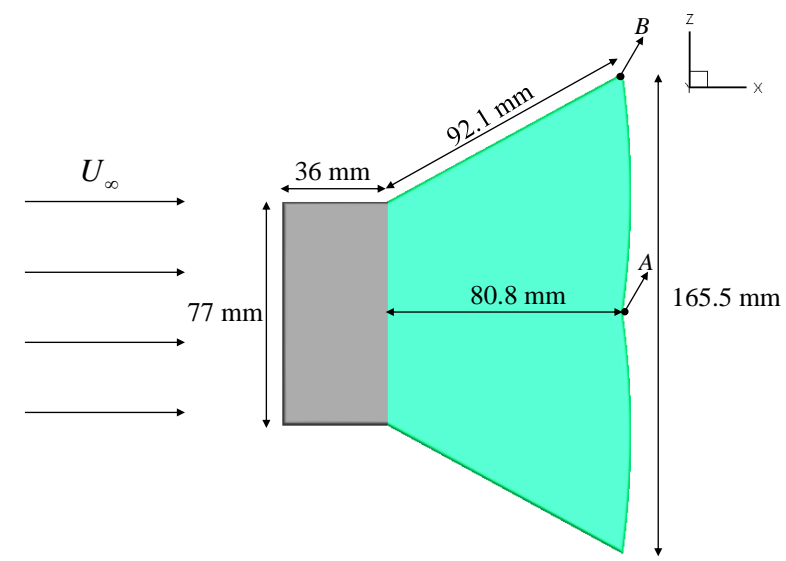

Flexible caudal fin

Fig. 1. Illustration of the fish peduncle-caudal model (a) and $x z$ view of the model and dimensions (b).

(a)

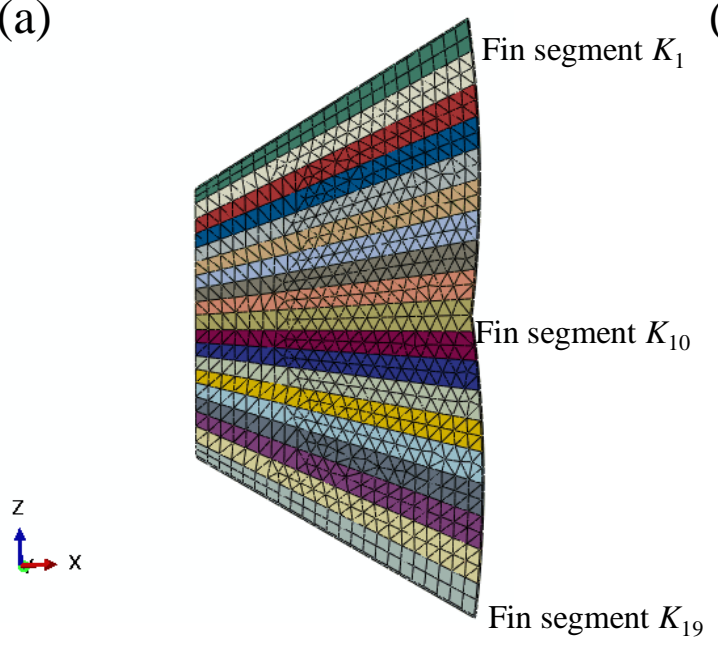

(b)

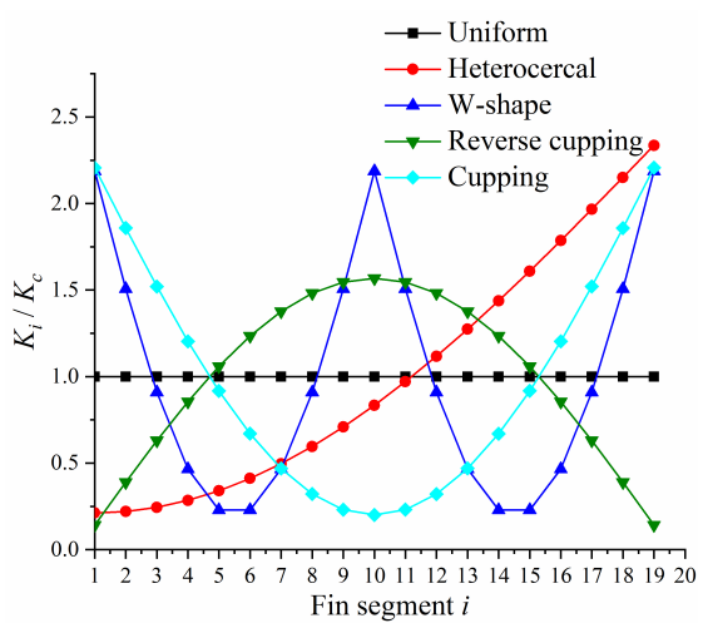

Fig. 2. The generated structural meshes of the fin with 19 segments in different colors (a) and the distribution patterns of stiffness (b). 


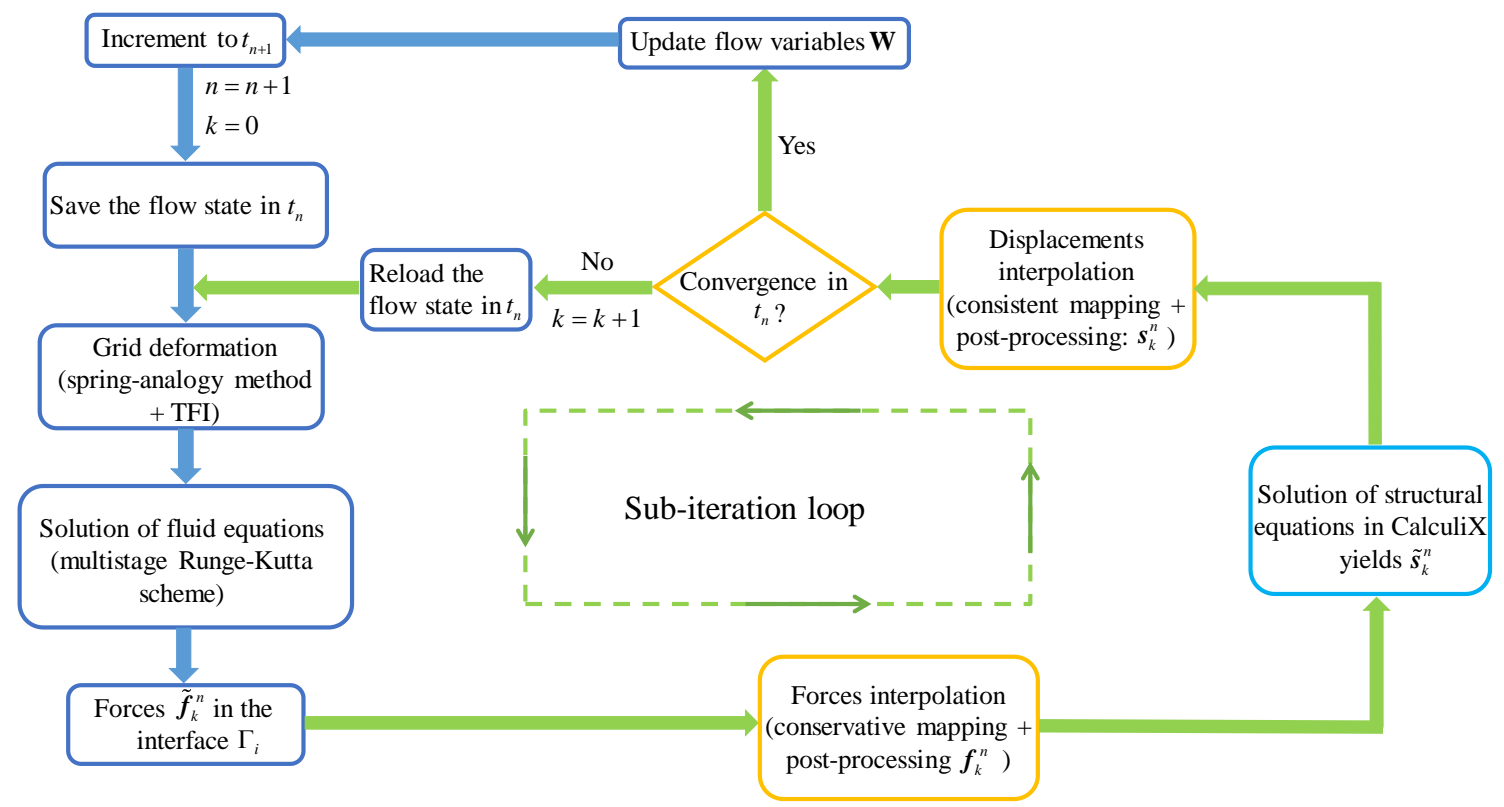

Fig. 3. Flow chart of the implicit FSI coupling in a partitioned approach.

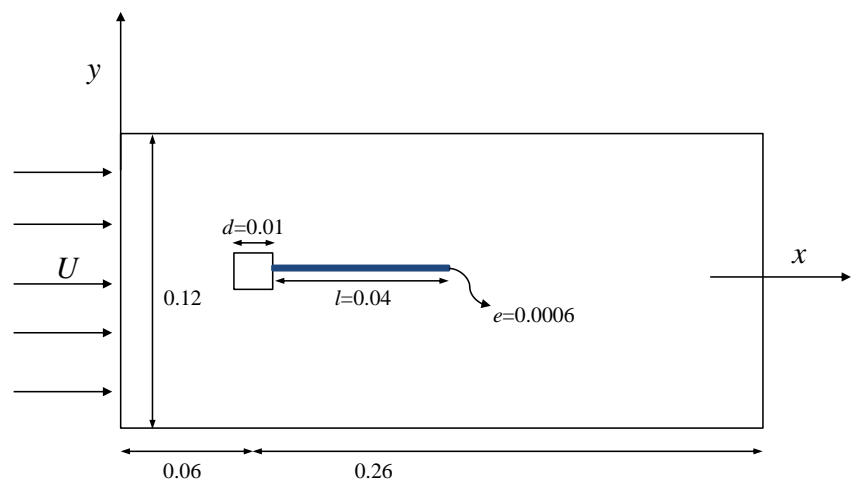

(a)

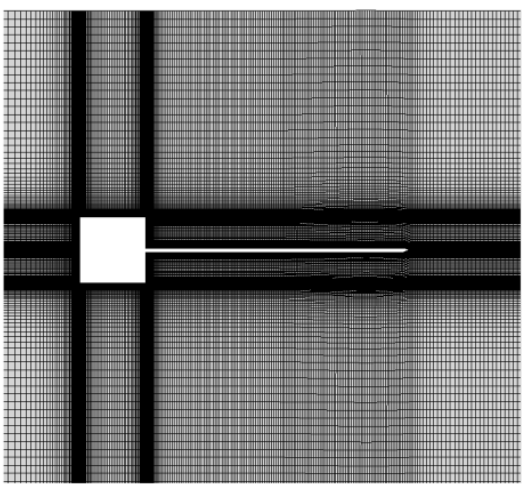

(b)

Fig. 4. Computational domain layout (a) and generated mesh (b) of a flexible cantilever behind a square cylinder. 


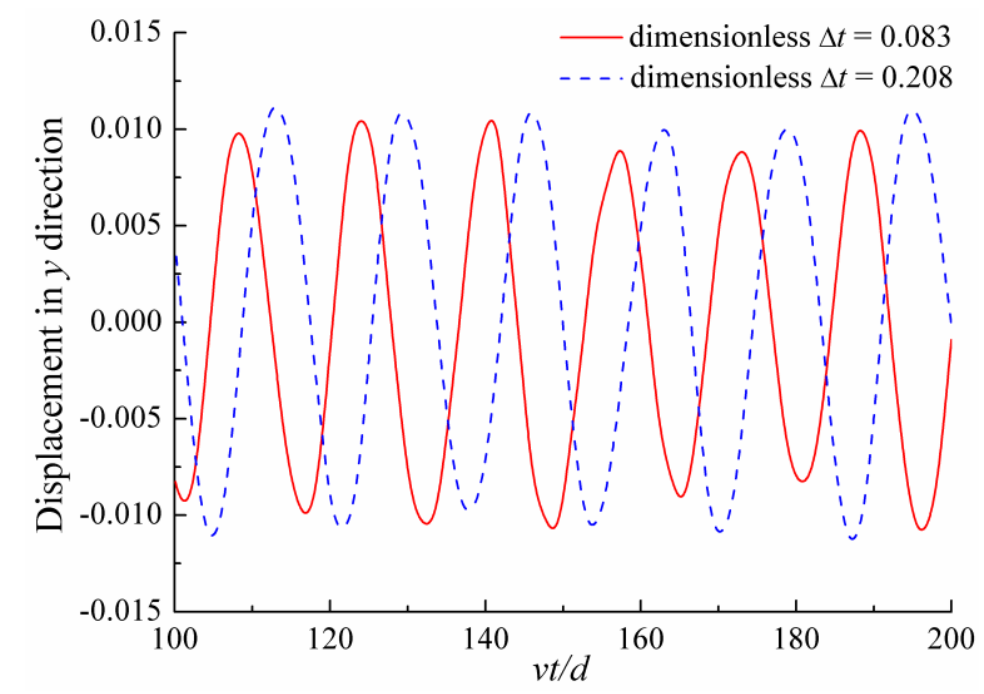

Fig. 5. Vertical tip displacement of the cantilever beam with different time step sizes.
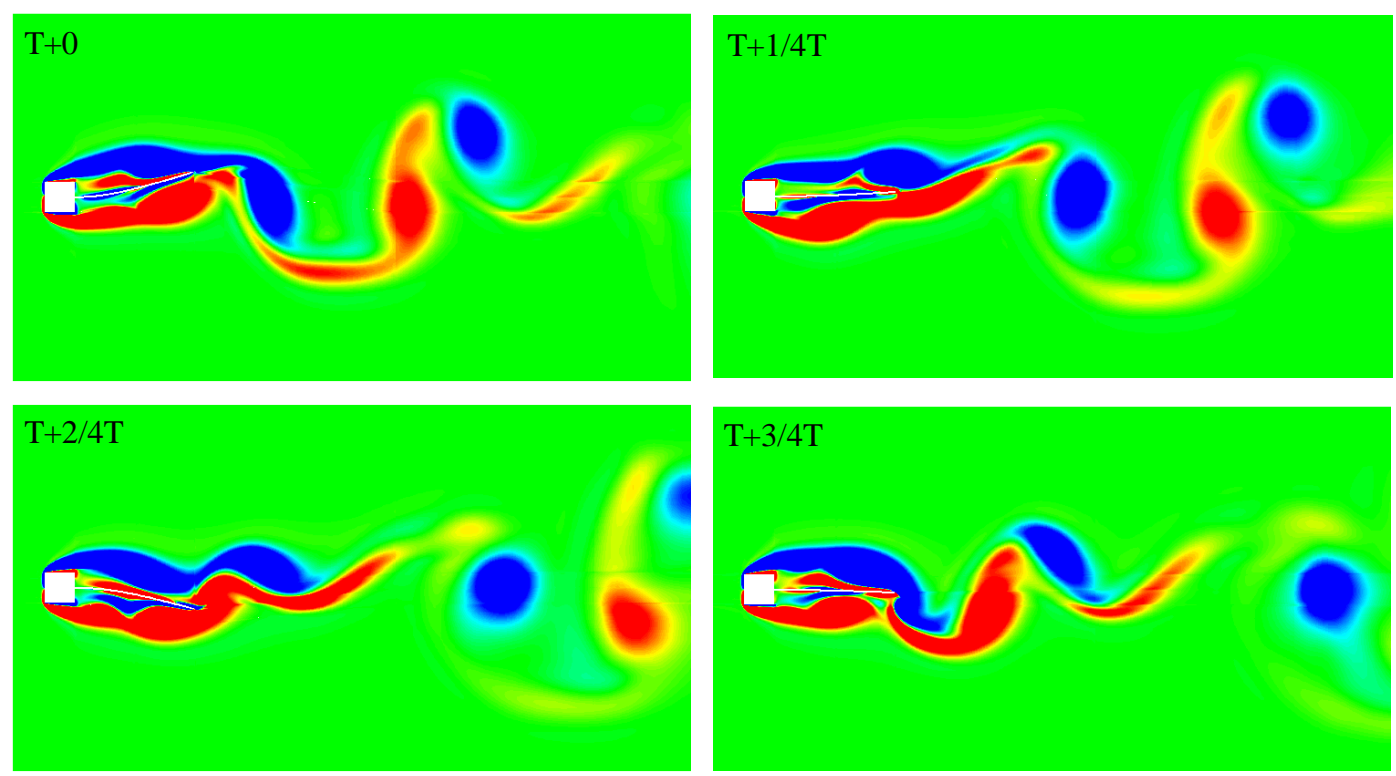

Fig. 6. Evolution of vorticity in $z$-direction around the cantilever within one oscillation period. 


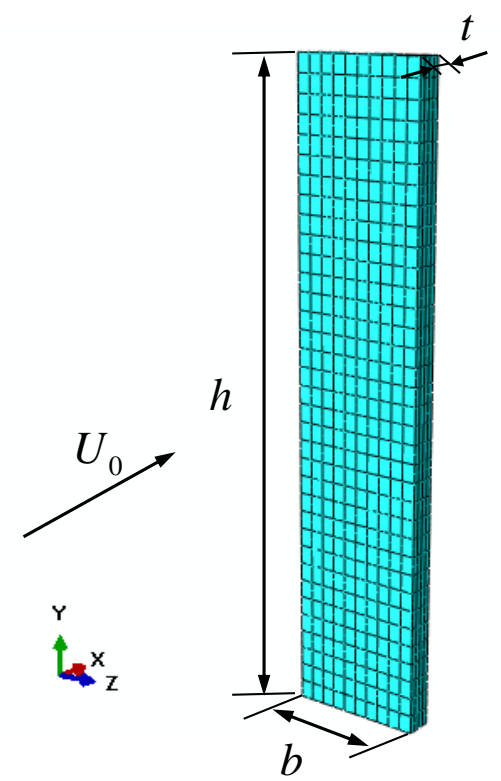

Fig. 7. The layout of flow over an elastic plate.

(a)

$$
y=1.8 b
$$

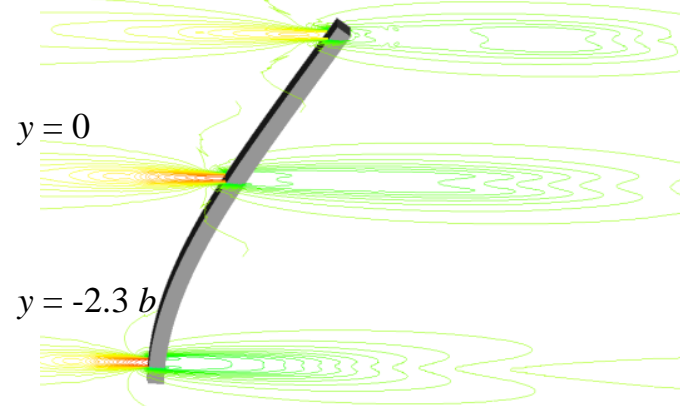

(b)

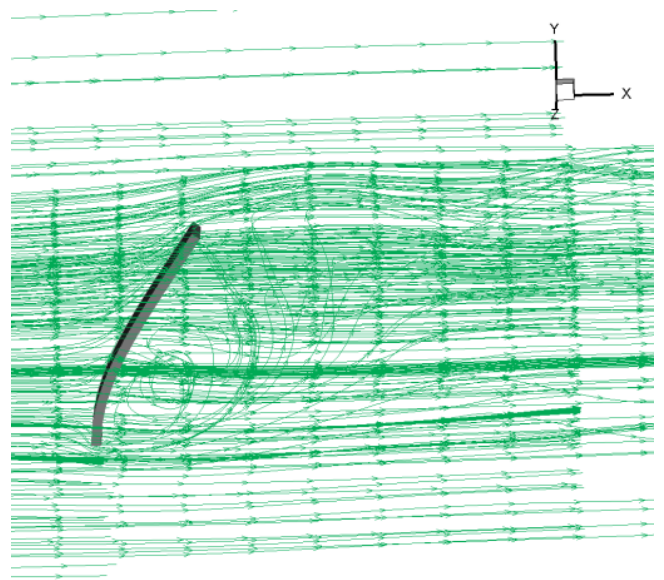

Fig. 8. The deformed plate with three representative horizontal slices colored with pressure distribution (a) and the streamline around the plate (b).

Table 1. Comparison of drag coefficient and deformation in the absence of gravity and buoyancy when $\mathrm{Re}=100$.

\begin{tabular}{cccc}
\hline & $C_{d}$ & $D_{x} / b$ & $D_{y} / b$ \\
\hline Tian et al. (2014) & 1.02 & 2.34 & 0.67 \\
Current study & 1.06 & 2.31 & 0.678 \\
\hline
\end{tabular}


Table 2. CFD mesh and time-step sensitivity test results.

\begin{tabular}{cccc}
\hline Categories & $\overline{C_{T}}$ & $\overline{C_{P}}$ & $\eta$ \\
\hline Mesh_Coarse, $\Delta t=0.00909$ & 0.221 & 1.045 & 0.211 \\
Mesh_Medium, $\Delta t=0.00694$ & 0.220 & 1.036 & 0.212 \\
Mesh_Fine, $\Delta t=0.00556$ & 0.220 & 1.033 & 0.213 \\
\hline
\end{tabular}

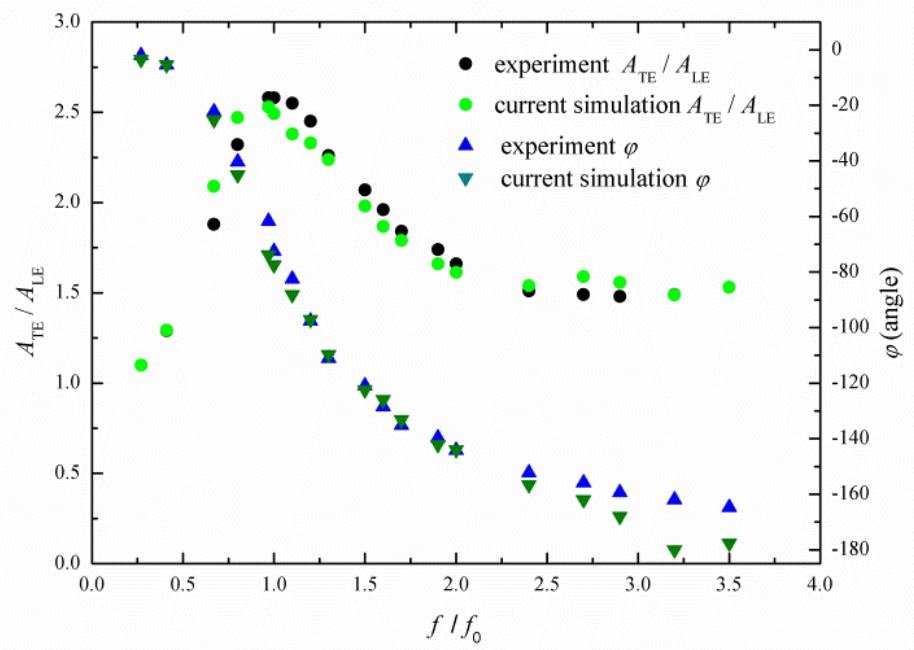

Fig. 9. The relative displacement of the trailing edge with respect to that of the leading edge of the flexible plate, $A_{\mathrm{TE}} / A_{\mathrm{LE}}$, and the corresponding phase shift as a function of the normalized frequency $f / f_{0}$ for $\operatorname{Re}$ $=6000, A_{\mathrm{TE}}=0.004 \mathrm{~m}$ and rigidity $\mathrm{B}=0.018 \mathrm{~N} \cdot \mathrm{m}$.

(a)

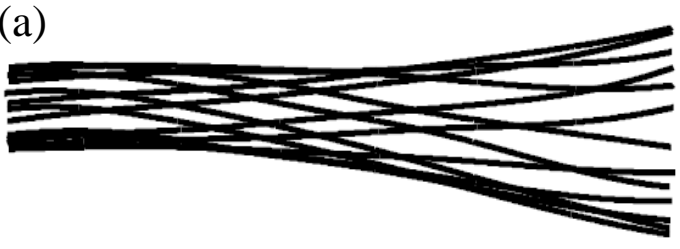

(b)

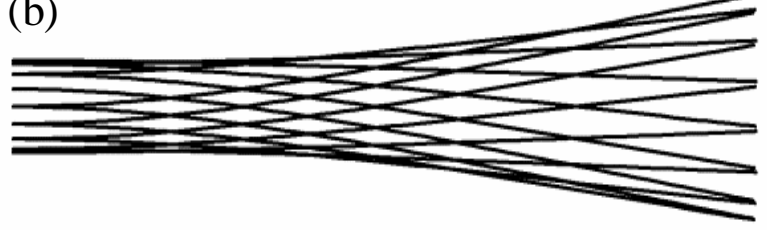

Fig. 10. Mode shape of the plate when $f / f_{0}=1$ obtained from the experiment (a) (Paraz et al., 2014) and current numerical simulation (b). 

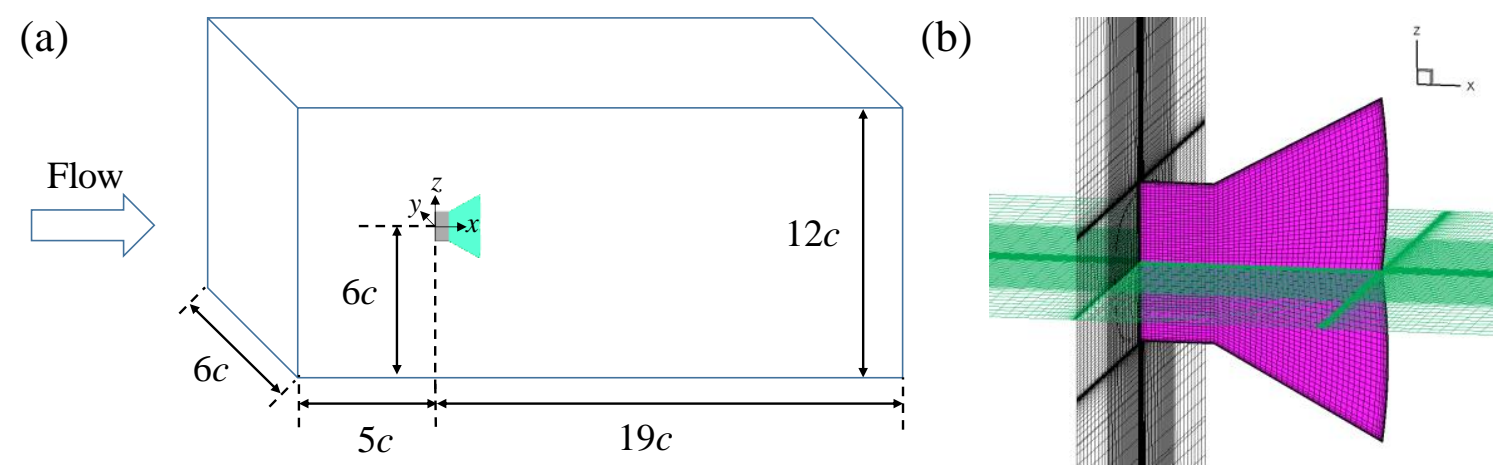

Fig. 11. Sketch of the computational domain (a) and the generated fluid mesh around the caudal pedunclefin model (b).

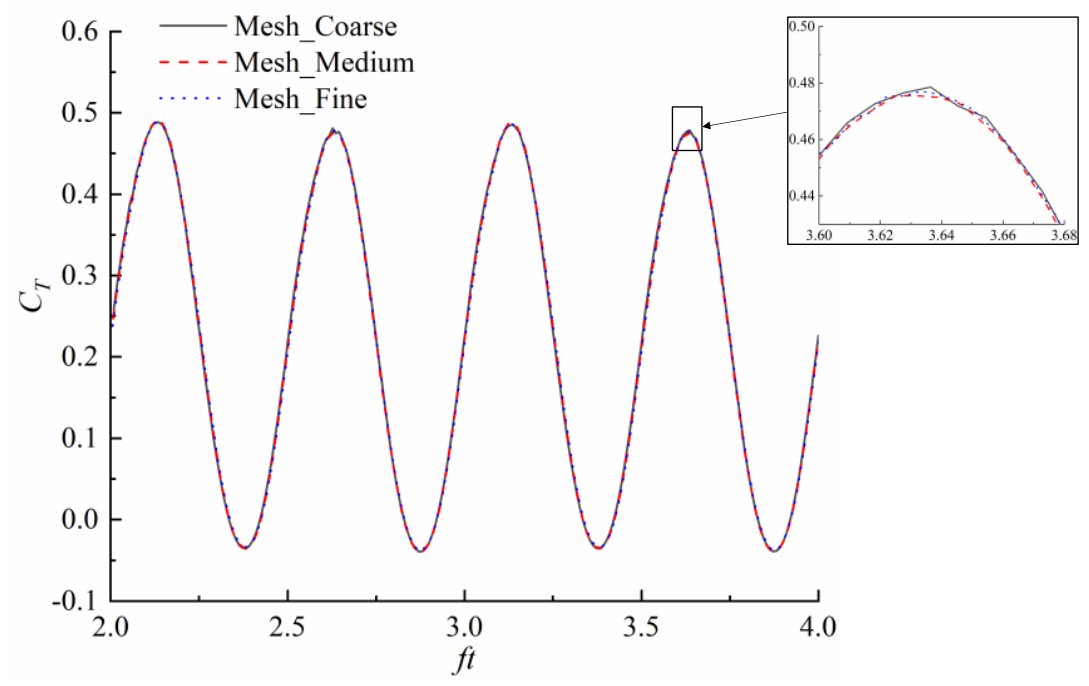

Fig. 12. Comparison of thrust coefficients $C_{T}$ with three different resolutions. 
(a)

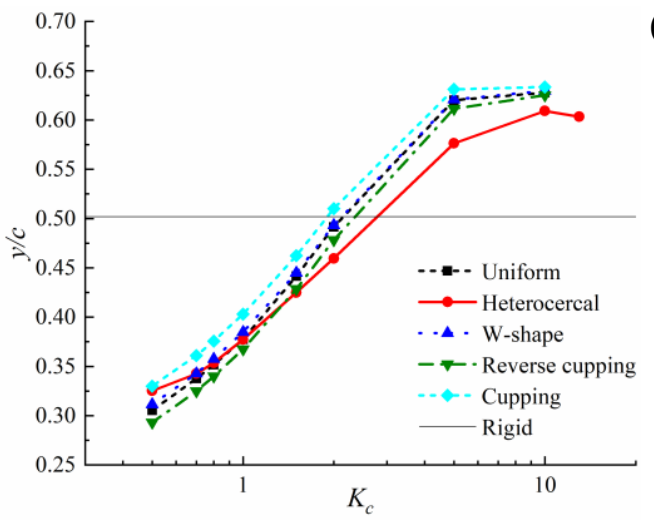

(c)

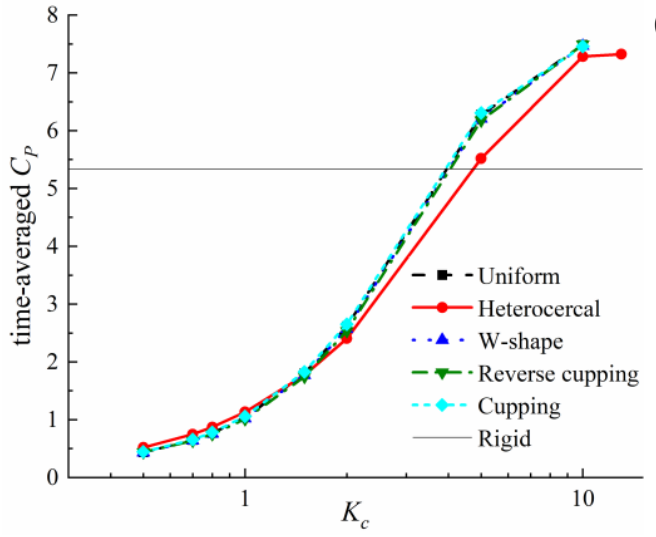

(e)

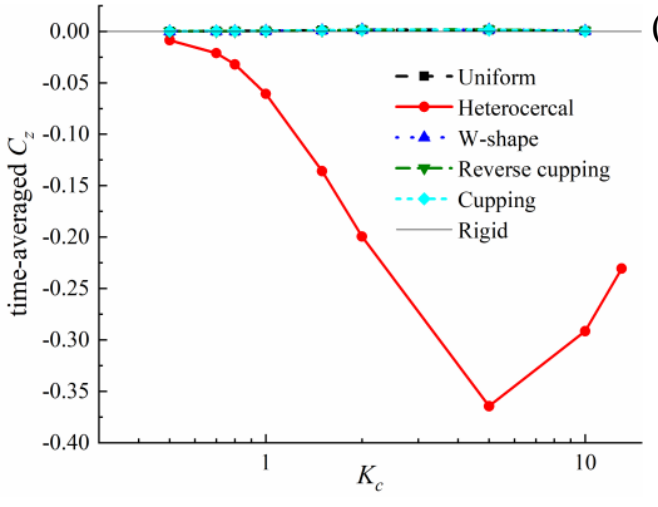

(b)

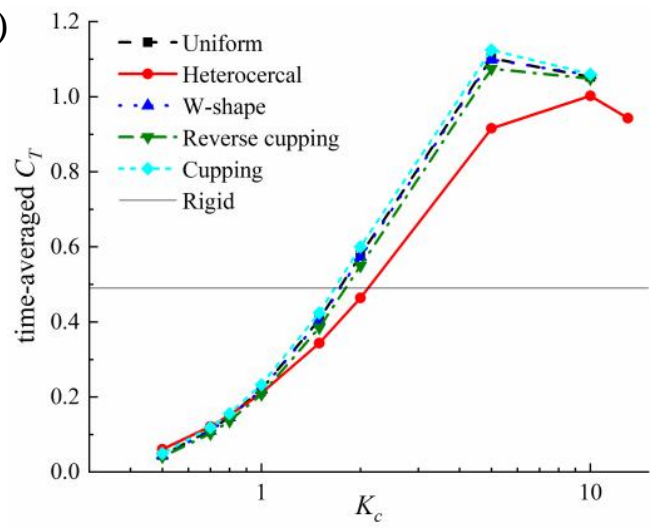

(d)

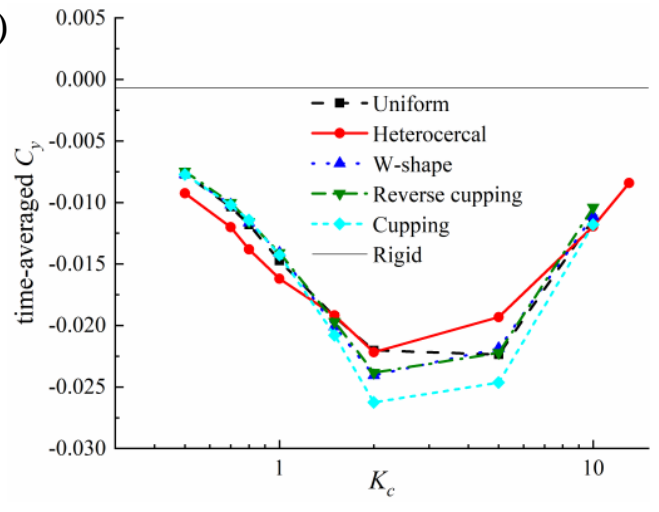

(f)

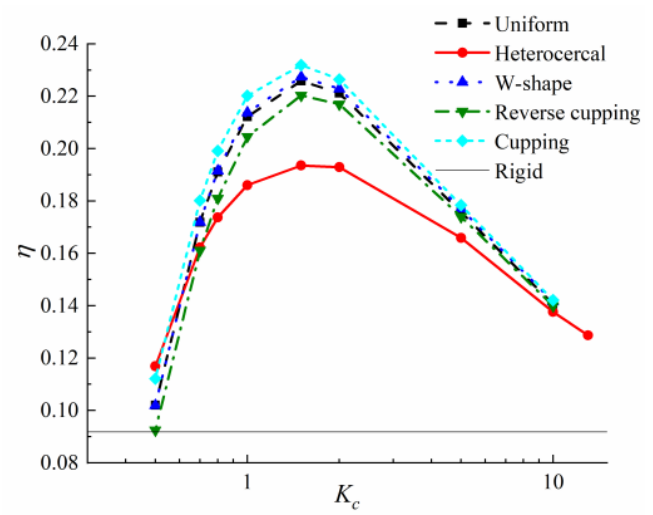

Fig. 13. Summary of the tail excursion at point $A$ (a), time-mean thrust (b), power input coefficients (b), lateral forces (d), lift forces (e) and propulsion efficiency (f) when flexural rigidities are varied for uniform (black dash), heterocercal (red solid), W-shape (blue dot), reverse cupping (olive dash dot) and cupping (cyan short dash) stiffness distribution. 
(a)

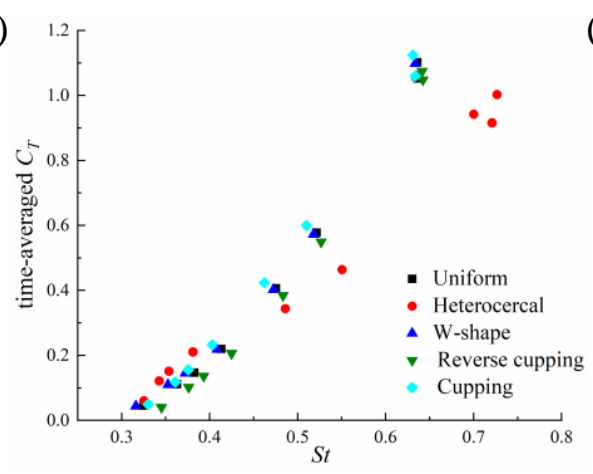

(b)

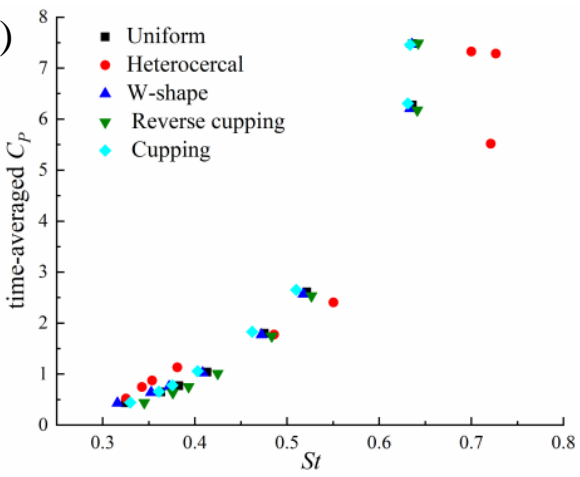

(c)

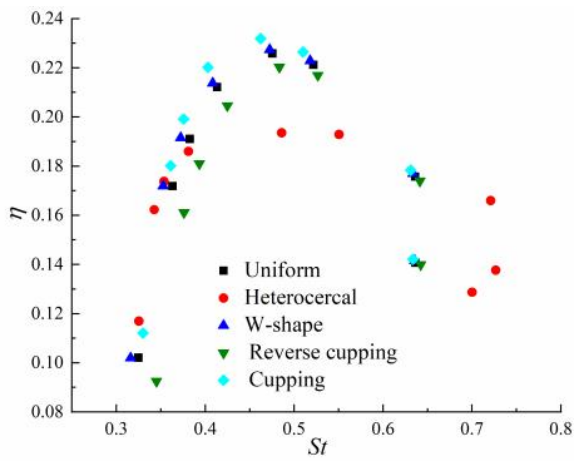

Fig. 14. The time-mean thrust (a), power input coefficients (b) and propulsion efficiency (c) re-plotted against the Strouhal number $S t$ defined using the tail excursion.
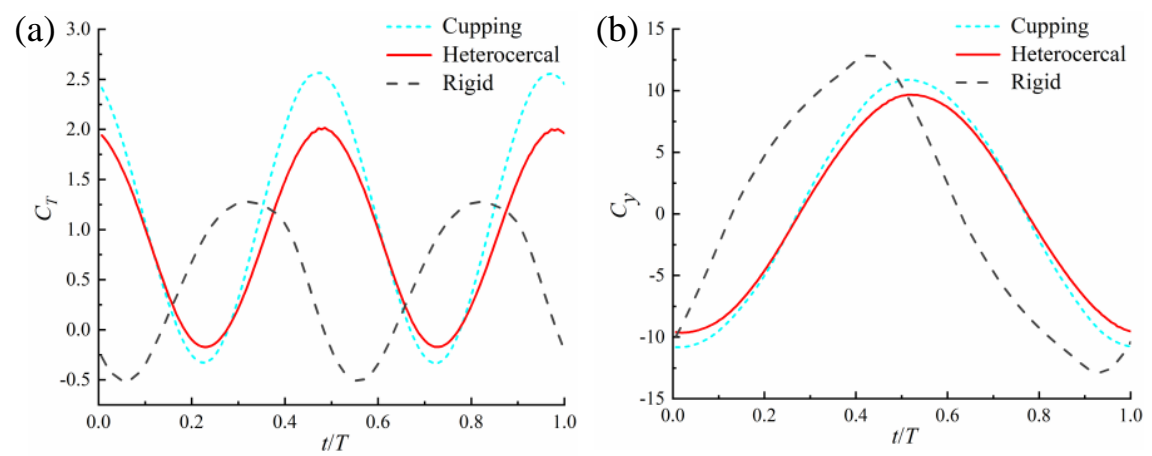

(c)

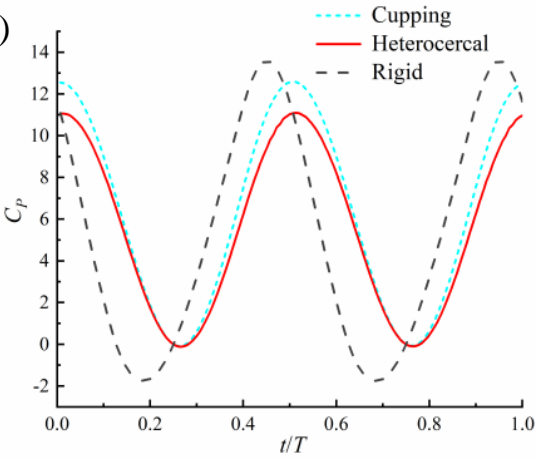

Fig. 15. Time history of $C_{T}, C_{y}$ and $C_{P}$ within one oscillation period of a fin with cupping (cyan short dash), heterocercal stiffness distribution (red solid) and a rigid fin (grey dark dash) when $K_{c}=5$. 


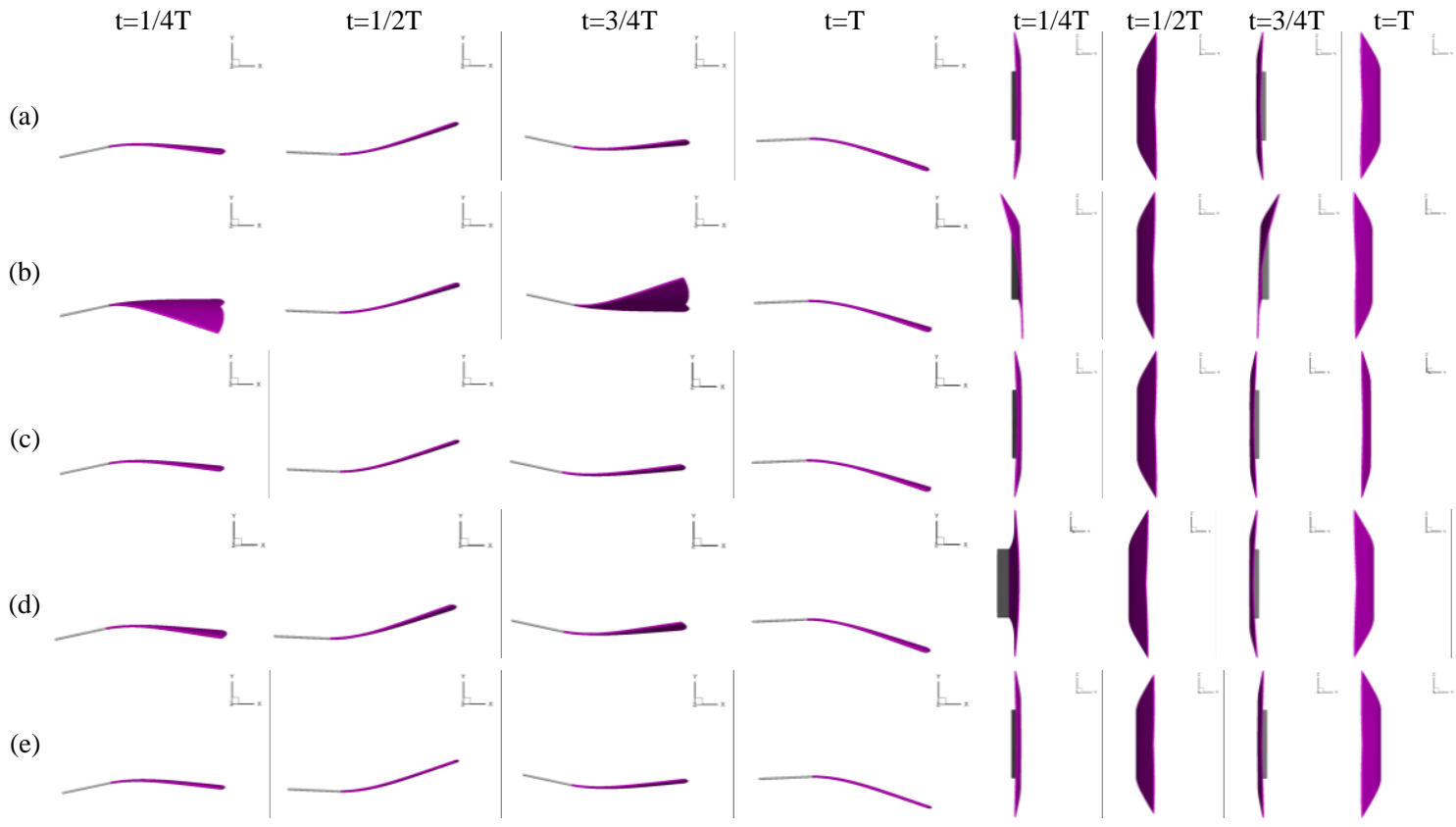

Fig. 16. Typical fin deformation in $x y$ (left column) and $y z$ view of fins with uniform (a), heterocercal (b), W-shape (c), reverse cupping (d) and cupping (e) stiffness profile. The fin is dyed in pink color for recognition. 


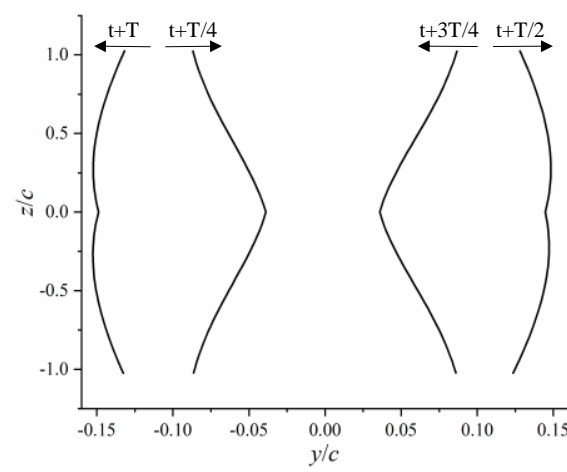

Uniform profile

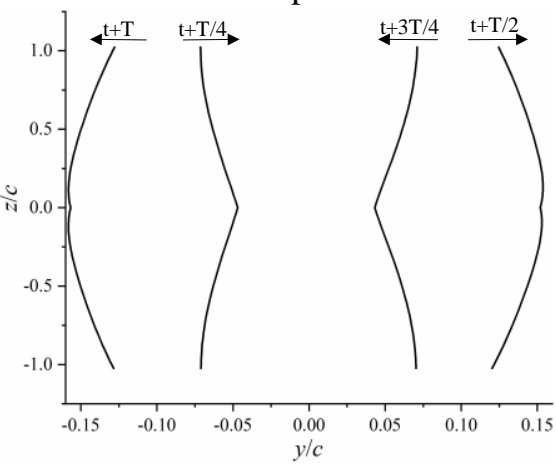

Cupping profile

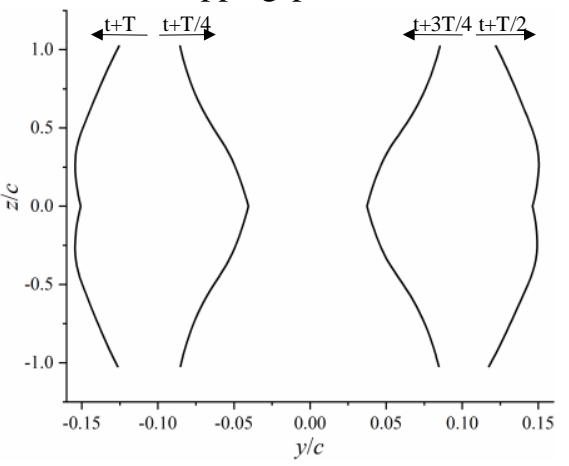

W-shape profile

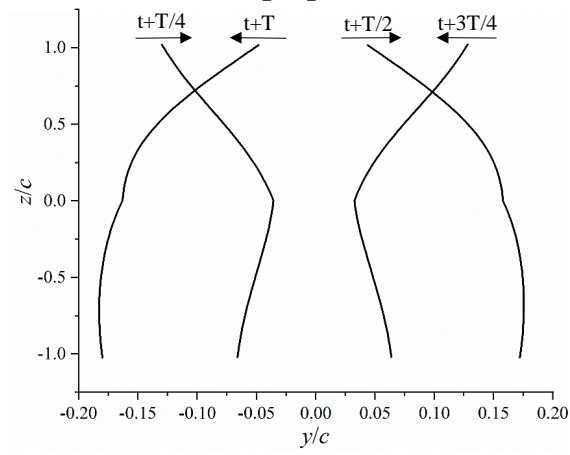

Heterocercal profile

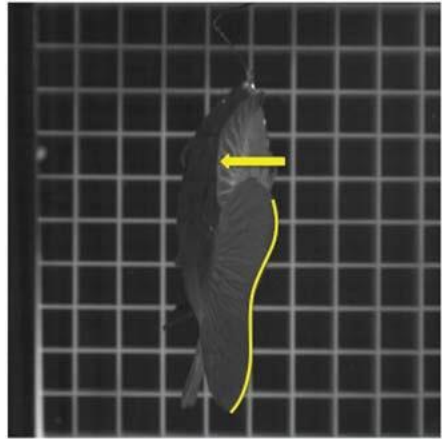

Flat motion

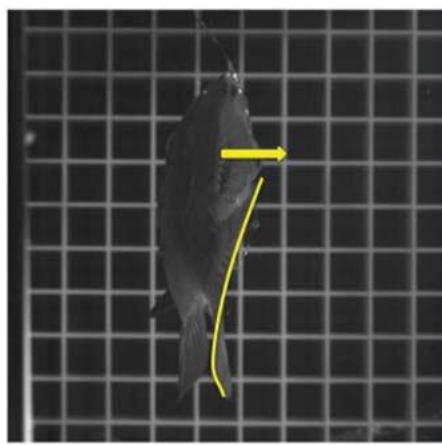

Cupping motion

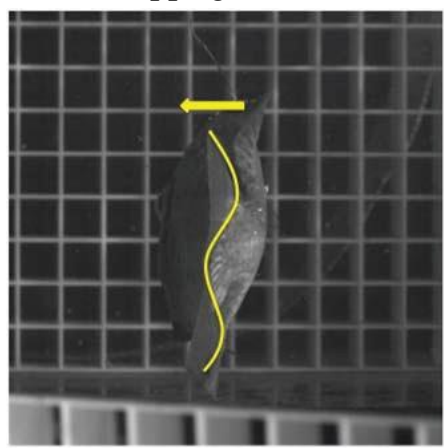

W motion

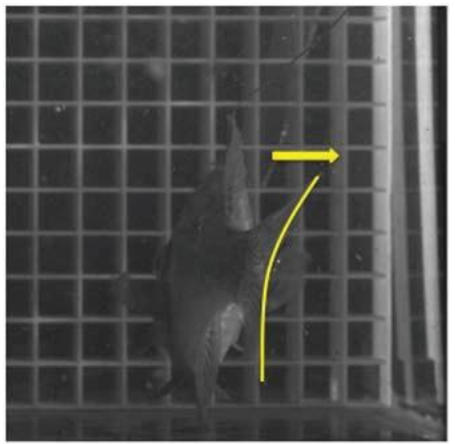

Rolling motion

Fig. 17. The deformation patterns of the trailing edge of the fin when $K_{c}=0.5$ (a), and posterior view of a bluegill fish adopted from (Esposito et al., 2012) (b). 
(a)

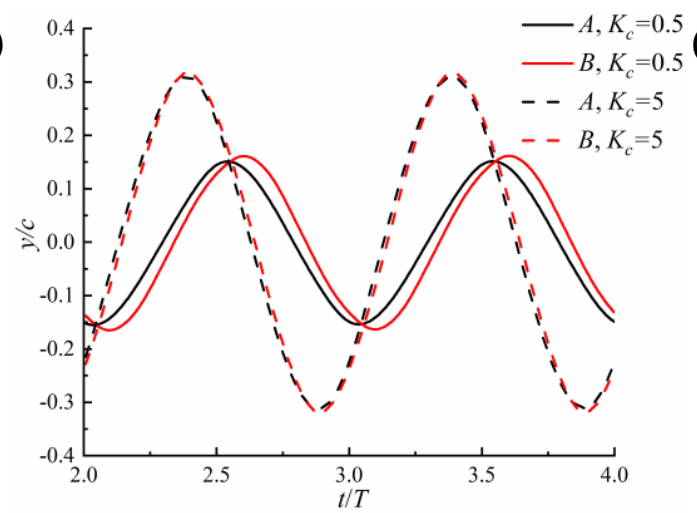

(c)

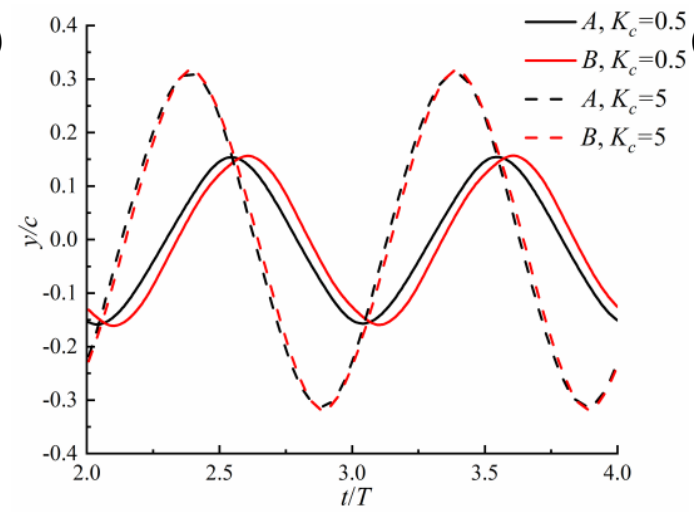

(b)

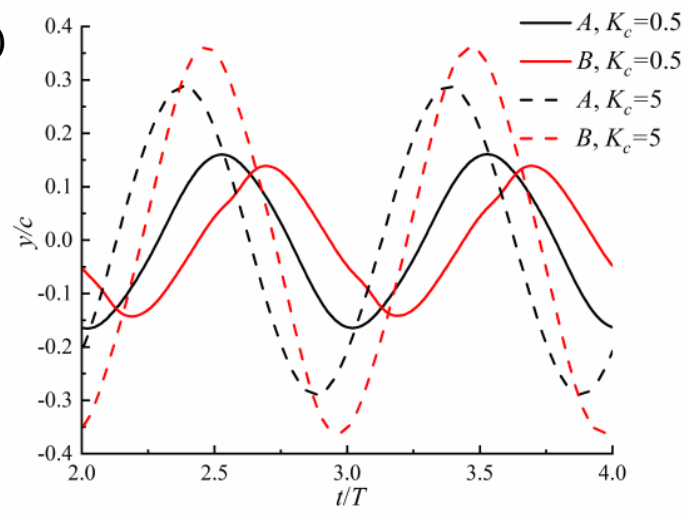

(d)

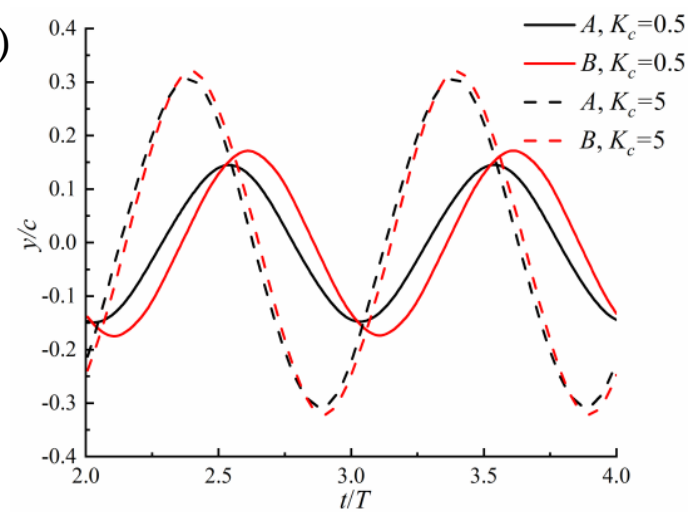

(e)

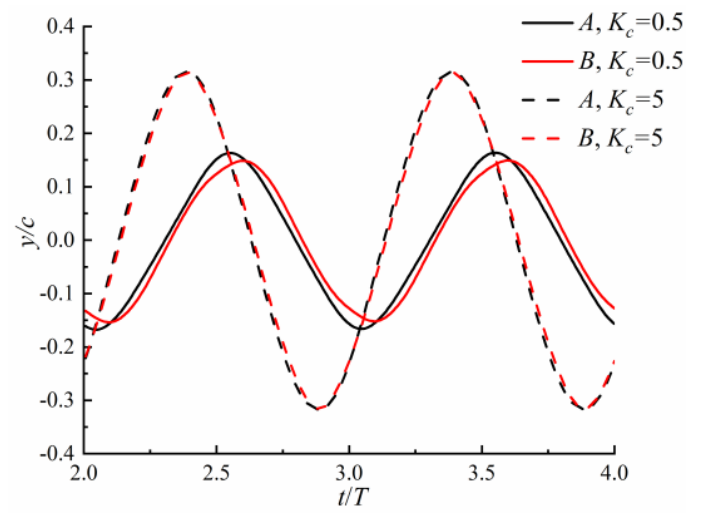

Fig. 18. The displacements of the point A and B when $K_{c}=0.5$ and $K_{c}=5$ for the fin with uniform (a), heterocercal (b), W-shape (c), reverse cupping (d) and cupping (e) stiffness distribution. 

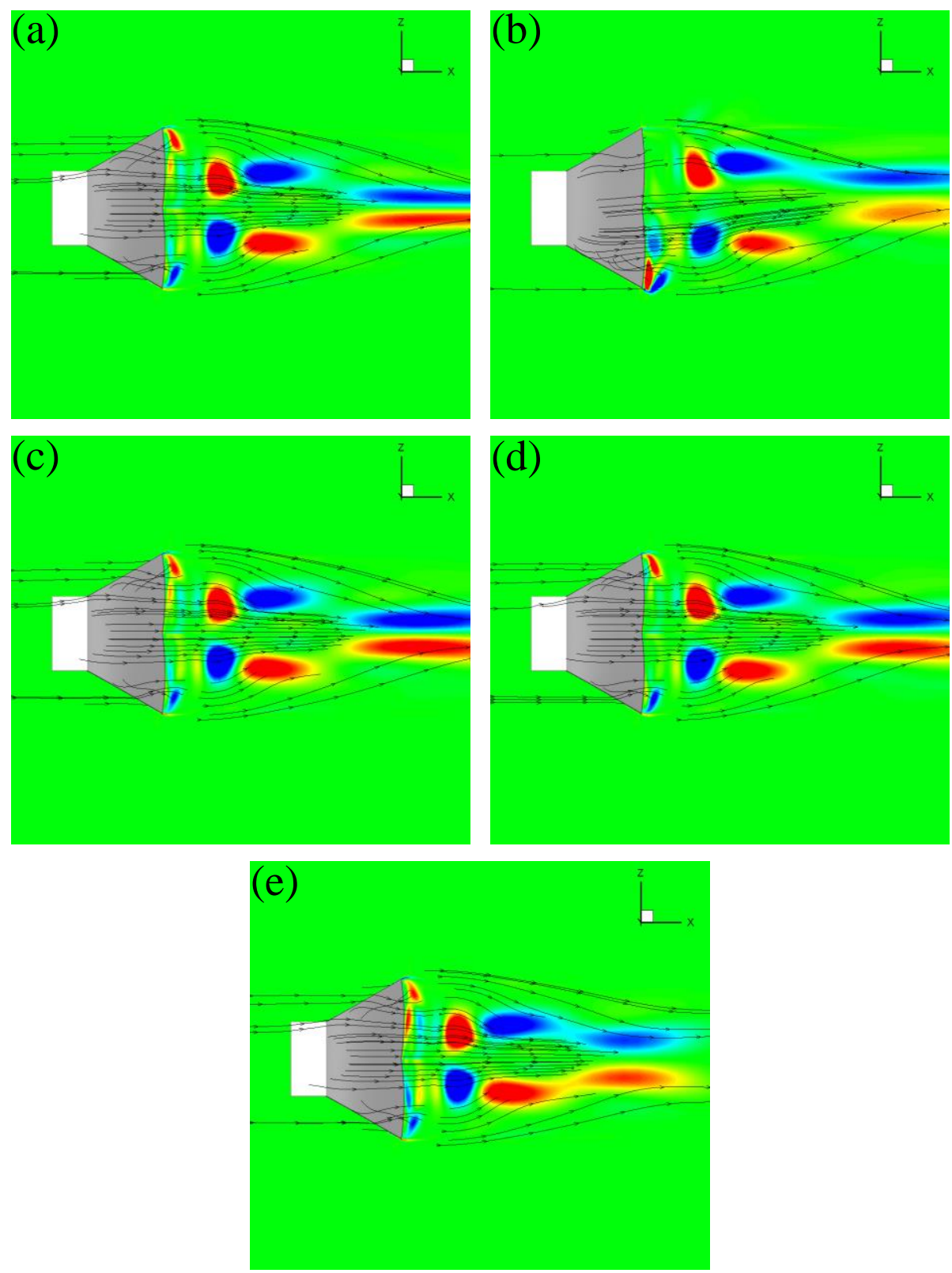

Fig. 19. The wake flow contoured in $Y$ vorticity along with streamlines at plane $y=0.3 c$ when $K_{c}=2$ at $t=T$ of the flexible fin with uniform (a), heterocercal (b), W-shape (c), reverse cupping (d) and cupping (e) stiffness distribution. 


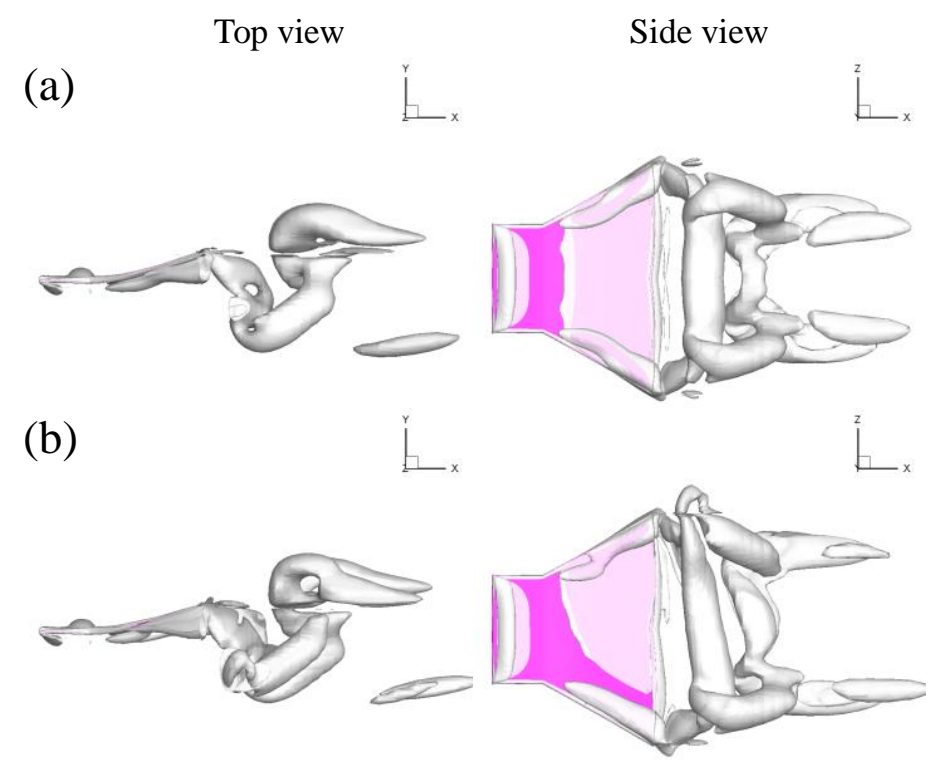

Fig. 20. Iso-surface of vorticity field ( $\mathrm{Q}$ criterion) when $K_{c}=2$ at $t=T$ in the wake of the flexible fins with cupping (a) and heterocercal (b) stiffness distribution. The peduncle-fin is dyed in pink color for recognition. 
(a)

(b)
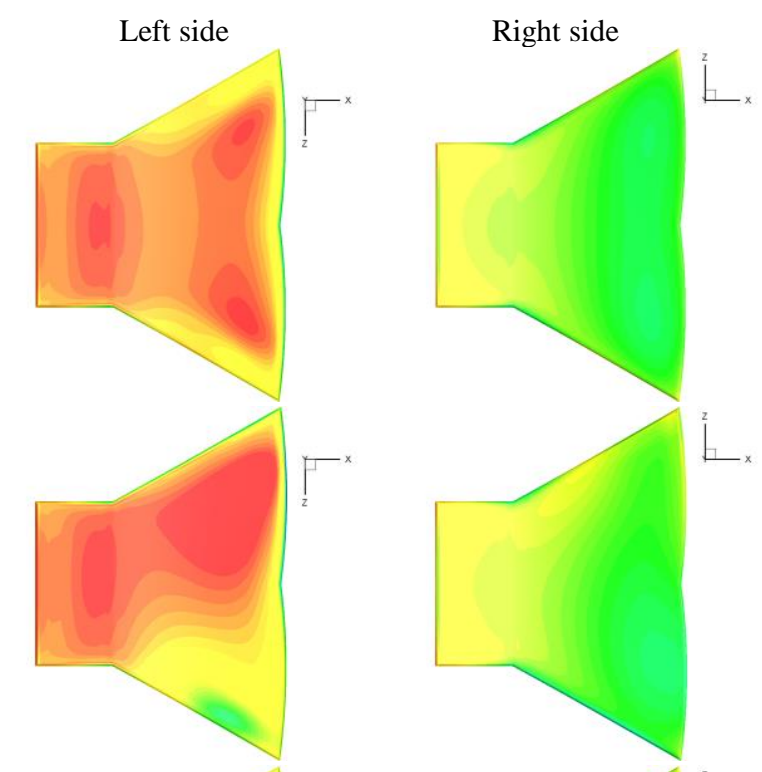

(c)

(d)
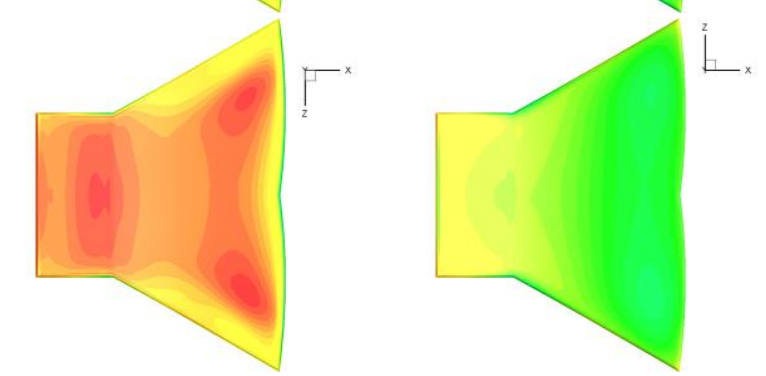

(e)
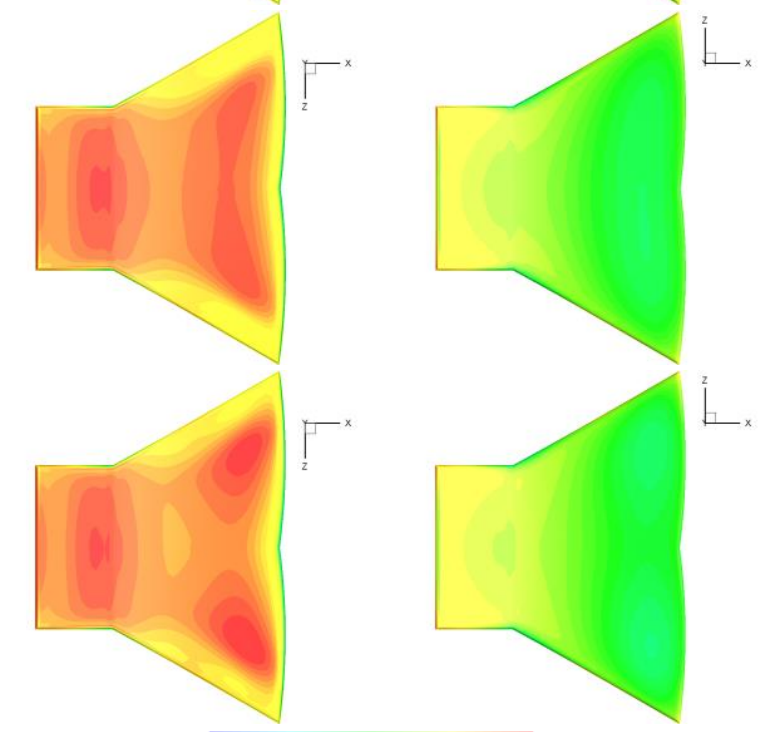

$\begin{array}{llllll}-11.0 & -8.5 & -6.0 & -3.5 & -1.0 & 1.5\end{array}$

Fig. 21. The distribution of the pressure coefficient $C_{\text {pressure }}=p / 0.5 \rho_{f} U_{\infty}^{2}$ on the left and right side of the surface of the caudal peduncle-fin when $K_{c}=2$ at $t=T$ of the flexible fin with uniform (a), heterocercal (b), $\mathrm{W}$-shape (c), reverse cupping (d) and cupping (e) stiffness distribution. The left and right are defined from the viewpoint at the posterior. 